\title{
THE THEOREMS OF BETH AND CRAIG IN ABSTRACT MODEL THEORY. I. THE ABSTRACT SETTING
}

BY

J. A. MAKOWSKY AND S. SHELAH

$$
\text { 1 שנינו ביחד וכל אחד לחוד }
$$

\begin{abstract}
In the context of abstract model theory various definability properties, their interrelations and their relation to compactness are investigated.
\end{abstract}

Introduction. This is the first of three papers on the analogues of the theorems of Beth and Craig of first order logic in abstract model theory. They grew out of an unpublished preprint [MS] ${ }^{2}$ which was revised and extended several times by results of both authors as well as other people. They unify results due to Badger, Ebbinghaus, Friedman, Gostanian, Gregory, Hrbacek, Hutchinson, Kaufmann, Magidor, Malitz, Makkai, Makowsky, Paulos, Shelah and Stavi.

In this paper we present the abstract setting; we suppose that the reader is familiar with a standard text on model theory such as [BS] and [CK], with Barwise's [Ba1] and [MSS]. The main results here are that:

Beth's theorem together with a Feferman-Vaught theorem for tree-like sums implies a weak form of Robinson's consistency lemma (5.4) and the Robinson consistency lemma together with the Feferman-Vaught theorem for pairs implies full compactness (6.2).

In [MS2] and [MS3], the continuations of the present paper, we present applications of the general theory to particular logics. [MS2] is devoted to compact logics and some new logics are introduced, and [MS3] is devoted to infinitary logics and $\Delta$-logics. Some complicated constructions are presented in detail there.

There are three aspects of Craig's interpolation theorem and its corollary, Beth's definability theorem:

(A) Philosophical. Every implicit definition is equivalent to an explicit definition.

Received by the editors July 13, 1976 and, in revised form, March 31, 1978, May 25, 1978 and September 15, 1978.

AMS (MOS) subject classifications (1970). Primary 02B20, 02B25, 02H10, $02 \mathrm{H} 13$.

${ }^{1}$ Cf. [Am].

${ }^{2}$ This has been circulating since Spring, 1975. 
(B) Mathematical. Although mathematicians never really cared whether they use explicit or implicit definitions, Beth's theorem tries to explain why we always have an explicit definition of a concept whenever it is uniquely describable. The ordering in real closed fields, e.g., is unique and the proof of this gives us an explicit definition of the ordering. Or formally real fields, where every element or its inverse can be represented as a sum of squares, can be uniquely ordered. Again the proof gives us an explicit definition. Another example: Uniquely orderable groups can be characterized by an $L_{\omega_{1} \omega}$ sentence (cf. Bludov [B1]) so again there must exist an explicit definition of this ordering in $L_{\omega, \omega}$. Since most proofs of Craig's theorem are effective (in the finitary case, in the infinitary case this is more complicated) the explicit definition can also be obtained on a purely syntactical level. But we do not know of any example where this observation gives us anything deeply mathematical.

The main motivation for this paper, however, stems from the third aspect:

(C) Metamathematical. Craig's theorem holds for predicate logic and second order logic as well as for $L_{\omega_{1} \omega}$ and $L_{A}$ where $A$ is an increasing union of countable admissible sets. First order logic can be characterized in terms of maximality with respect to some model theoretic properties. Now most of the proofs of such maximality theorems also give a proof of Craig's theorem. So "maximality" implies "Craig's theorem" sounds like a nice motto. Unfortunately $L_{A}$ does not seem to fit into this picture. More generally we do not know if there is a maximal logic satisfying the Löwenheim-Skolem theorem down to $\omega$ and Craig's theorem; in particular Harrington and Kunen have shown independently that $L_{\omega_{1} \omega}$ is not maximal in a very strong sense. Kunen uses $\mathrm{CH}$ and gets explicit extensions of $L_{\omega_{1} \omega}$ adding propositional connectives. Harrington gives an existence proof of $2^{2 \omega}$-many other connectives $P$ such that $L_{\omega_{1} \omega}(P)$ shares most of the properties of $L_{\omega_{1} \omega}$ without $\mathrm{CH}$. Both results are presented in [Ha].

Other aspects of maximality in connection with definability properties are discussed in Feferman [Fe1] and Stavi [St1].

The aim of these papers is twofold: To provide the reader with a reasonably complete picture of what logics do or do not satisfy what kind of definability theorems, providing proofs, counterexamples or references, and to discuss definability theorems in the setting of abstract model theory. The two cannot be separated completely. Some abstract theorems give us hints of how to find counterexamples. Some constructions of counterexamples split into two different parts one of which can be easily captured as an abstract theorem. What we hope to show, too, is how this interplay works. In detail this paper is organized as follows.

In $\$ 1$ compactness and Löwenheim-Skolem properties are defined as well 
as the Karp-property. Some theorems relating these properties are studied or quoted for later use.

In $\$ 2$ the interpolation and definability properties are introduced for pairs of logics: Craig's interpolation theorem, Beth's definability theorem, a weakened version of Beth's theorem. $\Delta$-interpolation and variations around Robinson's consistency theorem. Their mutual logical interdependence is exhibited, and a theorem of Barwise is improved (Theorem I.2.4 and its corollaries). Also a new characterization of predicate calculus is given as the maximal Karp-logic which satisfies Robinson's consistency theorem (Theorem 2.14).

In \$3 Feferman-Vaught-type theorems are discussed for pairs of logics and various sum-like operations on many-sorted structures. Another characterization of predicate calculus is given involving the Löwenheim-Skolem theorem, the weakened form of Beth's theorem and a Feferman-Vaught theorem for pairs of structures (Theorem 3.3 and Corollary 3.2).

In $\$ 4$ we review briefly some results due to Feferman and Makowsky on uniform reduction which relates Feferman-Vaught-type theorems with definability theorems.

In $\$ 5$ we describe a construction whose main motivation is to prove not Beth when not Craig. For credit see there. Variations of this construction will appear also in [MS2] and [MS3]. Its main content is a way to get implicit definitions out of counterexamples for definability theorems. It also yields an abstract theorem: Beth's theorem and a strong form of a Feferman-Vaught theorem imply the weak Robinson consistency theorem (Theorem 5.4).

In $\$ 6$ we prove that under some weak assumption on set theory Robinson's consistency theorem ${ }^{3}$ implies full compactness. Although not surprising, this is a highly nontrivial theorem of abstract model theory and shows that with more effort more abstract theorems should be provable.

In $\$ 7$ we finally describe a generalization of an old trick to show that second order logic does not satisfy Beth's theorem, which has been worked out by Paulos and Burgess.

We conclude the paper with a survey of what logics do or do not satisfy what kind of definability property and an extensive bibliography.

There are many open problems left in the field: How can one construct explicitly logics with prescribed properties? We are still in the state of many, but scattered, examples, sometimes with rather well developed model theory (cf. [MS2], [Ke1], [Ma3] and [BKM] for $L(Q)$ and its extensions and [Ke2], [Ba2] for infinitary logic), but no coherent theory is in sight. Abstract model theory had two great impulses from Lindström and Barwise, but the more

\footnotetext{
${ }^{3}$ Together with a Feferman-Vaught theorem for pairs of structures.
} 
intriguing questions, the hard technical results, which give a field its living, seem to develop slowly. We hope to go an important step in this direction.

Here are some problems:

Problem 1. Is there any fully compact logic which satisfies Craig's theorem or is there any countably generated logic which satisfies Robinson's consistency theorem and which is different from (extending) predicate calculus?

Problem 2. What are the properties compact logics have in common? What conditions are needed to prove that the union of two compact logics is again compact (or similar for other properties than compactness)?

Kueker [Kue] has developed a general theory around the LöwenheimSkolem theorem. This is what we have in mind for an answer to Problem 2. A first step in such a direction has been made by Stavi [St2]. More explicit problems may be found in [Sh2] or in the remainder of this paper and in [MS2], [MS3].

Problem 3. What kind of partial isomorphisms generate reasonable logics? Is there a partial isomorphism relation (game characterization) for any elementary equivalence relation of an abstract logic? How do the partial isomorphisms reflect the properties of logics?

Stavi and Nadel [NSt], [Na] have some interesting work in this direction. An interesting test-case are the various Henkin-quantifiers discussed in [Wa] and [Ba4]. Very recently Caicedo [Ca] got very general results concerning Problem 3, part 1.

1. Karp-logics, compactness and cardinality of models. All the definitions of abstract model theory are taken from [Bal] unless otherwise stated. Note that our languages are mostly finite and all logics have the finite occurence property. Infinite languages occur in connection with diagrams. Universes are many sorted and PC-classes may admit extra universes, so reducts are relativized reducts. We let $L$ vary over languages and $L^{*}, L^{\dagger}$, etc. over logics. We use also $L^{*}$ for the $L^{*}$-formulas (sentences). ${ }^{4}$ Satisfaction is denoted by $\hat{F}_{L^{*}}$ or, when no confusion is possible, by $\vDash . L$-structures are denoted by $\mathfrak{X}, \mathfrak{B}$,

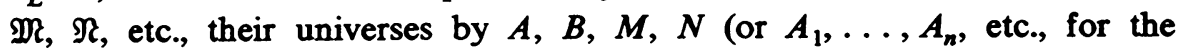
many-sorted case).

We denote by $\operatorname{Th}_{L^{*}}(\mathfrak{Q})=\left\{\varphi \in L^{*} \mid \mathfrak{A} \vDash \varphi\right\}$ and $\mathfrak{A} \equiv \mathfrak{B}\left(L^{*}\right)$ if $\operatorname{Th}_{L^{*}}(\mathfrak{X})=$ $\mathrm{Th}_{L^{*}}(\mathfrak{B})$. If $L^{*}$ and $L^{\dagger}$ are two logics, we write $L^{*}<_{\mathrm{Th}} L^{\dagger}$ if whenever $\mathfrak{A} \equiv \mathfrak{B}\left(L^{\dagger}\right)$ then also $\mathfrak{A} \equiv \mathfrak{B}\left(L^{*}\right)$.

Let $L^{*}$ be a logic. $L^{*}$ is said to have the Karp-property (is a Karp-logic) if for every $L$ and two $L$-structures $\mathfrak{A}, \mathfrak{B}, \mathfrak{U} \simeq_{p} \mathfrak{B}$ implies that $\mathfrak{A} \equiv \mathfrak{B}\left(L^{*}\right)$.

Here $\mathfrak{A} \simeq_{p} \mathfrak{B}$ means that there is a set of partial isomorphisms with the Back and Forth Property (cf. [Ba1]). By a theorem of Karp [Ba1] this is equivalent to $\mathfrak{X} \equiv \mathfrak{B}\left(L_{\infty \omega}\right)$.

${ }^{4}$ Note that $L$ is not always a set. 
THEOREM 1.1 (BARWISE). If $L^{*}$ is a Karp-logic then $L^{*}<_{\mathrm{Th}} L_{\infty}$.

A proof may be found in [Ba3].

THEOREM 1.2 (LINDSTRÖM/BARWISE). If $L^{*}$ is a Karp-logic in which $\langle\omega,\langle\rangle$ is not characterizable by a single sentence even with additional predicates, then $L^{*}=L_{\omega \omega}$.

A proof may be found in [Ba2] or [Li1], [Li2].

A logic $L$ is said to be $(\kappa, \lambda)$-compact $(\kappa, \lambda$ cardinals, $\kappa>\lambda)$ whenever given a set of sentences $\Sigma$ of $L^{*}$ of cardinality $\kappa$ such that each subset $\Sigma_{0}$ of $\Sigma$ of cardinality $<\lambda$ has a model, then $\Sigma$ has a model. Given two logics $L^{*}$ and $L^{\#}$ we write $L^{*}<L^{\#}$ if for every $\varphi \in L^{*}$ there is a $\varphi^{\prime} \in L^{\#}$ with the same models, $L^{*}=L^{\#}$ if $L^{*}<L^{\sharp}$ and $L^{\#}<L^{*}$.

Note that if $\kappa_{1}<\kappa$ and $\lambda_{1}>\lambda$ then $(\kappa, \lambda)$-compactness implies $\left(\kappa_{1}, \lambda_{1}\right)$ compactness.

Proposition 1.3. A logic $L^{*}$ is $(\omega, \omega)$-compact iff $\langle\omega,<\rangle$ is not characterizable by a countable set of sentences of $L_{1}^{*}$ for some $L_{1} \supset L$.

A proof may be found in [Ba2] or [F1].

Let $\lambda, \kappa, \mu$ be cardinals and $L^{*}$ a logic. We say that $L^{*}$ has the $(\lambda, \kappa)$ Löwenheim-Skolem property for sets of sentences of cardinality $\mu$ and write $\operatorname{LS}_{\mu}(\lambda, \kappa)$ if whenever $\Sigma$ is a set of sentences of $L^{*}$ of cardinality $\mu$ which has a model of cardinality $\lambda$ then it has a model of cardinality $\kappa$. If $\mu$ is finite we omit it. $\operatorname{LS}_{\mu}(\kappa)$ stands "for all $\lambda>\kappa \operatorname{LS}_{\mu}(\lambda, \kappa)$ ".

Proposition 1.4. Let $L^{*}$ be a fixed logic.

(i) If $\mu_{0}<\mu$ and $L^{*}$ has $\operatorname{LS}_{\mu}(\kappa, \lambda)$ then $L^{*}$ has $\operatorname{LS}_{\mu_{0}}(\kappa, \lambda)$.

(ii) If $L^{*}<L^{\dagger}$ and $L^{\dagger}$ is $(\kappa, \lambda)$-compact (has $\operatorname{LS}_{\mu}(\kappa, \lambda)$ ) so $L^{*}$ is $(\kappa, \lambda)$-compact (has $\operatorname{LS}_{\mu}(\kappa, \lambda)$ ).

THEOREM 1.5. (i) (Barwise) If $L^{*}$ has $\mathrm{LS}(\kappa, \omega)$ for all $\kappa \geqslant \omega$ then $L^{*}$ is a Karp-logic, provided $L$ is finite.

(ii) If $L^{*}$ has $\operatorname{LS}_{\omega}(\omega, \kappa)$ for some $\kappa>\omega$ then $L$ is $(\omega, \omega)$-compact.

(iii) (Lindström) If $L^{*}$ has $\operatorname{LS}(\omega, \kappa)$ for some $\kappa>\omega$ and $\operatorname{LS}(\omega)$ then $L^{*}=$ $L_{\omega \omega}$.

(iv) (Lindström) If $L^{*}$ is $(\omega, \omega)$-compact and has $\mathrm{LS}(\omega)$ then $L=L_{\omega \omega}$.

Proofs of (i) may be found in [Ba1] as well as for (ii), (iii) and (iv) follow using Theorem 1.2.

Shelah showed that $\operatorname{LS}(\kappa, \omega)$ does not imply $\operatorname{LS}_{\omega}(\kappa, \omega)$. A detailed study of LS $(\omega)$ may be found in [Kue].

The following is an easy exercise and will be used later. 
Proposition 1.6. If a logic $L^{*}$ staisfies $\mathrm{LS}(\lambda)$ then it has up to logical equivalence at most $2^{2^{\lambda}}$-many sentences and therefore almost $2^{2^{2^{\lambda}}}$-many complete theories.

2. Definability and interpolation properties. Let $L^{*}$ be a logic, $K$ a class of $L$-structures, and $\varphi \in L^{*}$. We define

$$
\operatorname{Mod}(\varphi)=\{\mathfrak{U} \mid \mathfrak{U} \text { is an } L \text {-structure and } \mathfrak{U} \vDash \varphi\}
$$

If $L_{0} \subset L$,

$\operatorname{Mod}(\varphi) \uparrow_{L_{0}}=\left\{\mathfrak{U} \mid \mathfrak{A}\right.$ is an $L_{0}$-structure which has an expansion

to an $L$-structure $\left.\mathfrak{U}^{\prime} \vDash \varphi\right\}$,

$$
\begin{aligned}
& K \in \operatorname{EC}\left(L^{*}\right) \text { iff } K=\operatorname{Mod}(\varphi) \text { for some } \varphi \in L^{*}, \\
& K \in \operatorname{PC}\left(L^{*}\right) \text { iff } K=\operatorname{Mod}(\varphi) \uparrow_{L} \text { for some } \varphi \in L_{1}^{*}, L_{1} \supset L .
\end{aligned}
$$

Interpolation properties. Let $L^{*}$ and $L^{\dagger}$ be two logics, $L^{*}<L^{\dagger}$. We say CRAIG $\left(L^{*}, L^{\dagger}\right)$ holds if whenever $K_{1}, K_{2} \in \operatorname{PC}\left(L^{*}\right)$ and $K_{1} \cap K_{2}=\varnothing$ then there is $K_{3} \in \mathrm{EC}\left(L^{\dagger}\right)$ such that $K_{1} \subseteq K_{3}$ and $K_{3} \subseteq \bar{K}_{2}\left(\bar{K}_{2}\right.$ the complement of $K_{2}$ with respect to $L$-structures).

We say $\Delta-\operatorname{Int}\left(L^{*}, L^{\dagger}\right)$ holds if whenever $K_{1}, K_{2} \in \mathrm{PC}\left(L^{*}\right), K_{1} \cap K_{2}=\varnothing$ and $K_{1} \cup K_{2}=\operatorname{str}(L)$ (= all $L$-structures) then $K_{1}=\bar{K}_{2} \in \operatorname{EC}\left(L^{\dagger}\right) . \Delta\left(L^{*}\right)$ is the smallest extension $L^{\dagger}$ of $L^{*}$ such that $\Delta-\operatorname{Int}\left(L^{*}, L^{\dagger}\right)$ holds. (For its existence and construction, cf. [MSS].)

Definability properties. We say that $\operatorname{BETH}\left(L^{*}, L^{\dagger}\right)\left(\operatorname{WBETH}\left(L^{*}, L^{\dagger}\right)\right)$ holds, if given $\varphi \in L_{1}^{*}, L_{1}=L \cup\{P\}, P$ an $n$-ary predicate symbol, every $L$-structure has at most (exactly one) expansion $\langle\mathfrak{A}, P\rangle \vDash \varphi$; then

$$
\left\{\langle\mathfrak{A}, \bar{a}\rangle \mid \mathfrak{A} \text { has an expansion }\langle\mathfrak{A}, P\rangle \vDash \varphi \text { and } \bar{a} \in P^{\mathfrak{x}}\right\} \in \mathrm{EC}\left(L^{\dagger}\right) \text {. }
$$

Joint consistency. $T \subseteq L^{*}$ is $L^{*}$-complete if whenever $T$ is a set, $\mathfrak{A} \vDash T$ and $\mathfrak{B} \vDash T$ then $\mathfrak{A} \equiv \mathfrak{B}\left(L^{*}\right)$. We say that $\operatorname{ROB}\left(L^{*}, L^{\dagger}\right)\left(\mathrm{WROB}\left(L^{*}, L^{\dagger}\right)\right)$ holds if whenever $T \subseteq L^{\dagger}$ is $L^{\dagger}$-complete, $L_{1}=L \cup\{P\}, P$ an $n$-ary predicate symbol, and $\varphi, \psi \in L_{1}^{*}$ are such that $T \cup\{\varphi(P)\}$ and $T \cup\{\psi(P)\}$ have a model, then $T \cup\left(\left\{\varphi(P), \psi\left(P^{\prime}\right)\right\}\right)\left(\left\{\varphi(P), \psi\left(P^{\prime}\right)\right\}\right)$ has a model (where $P^{\prime}$ is a new $n$-ary predicate symbol not in $L_{1}$ and $\psi\left(P^{\prime}\right)$ is the result of substituting $P^{\prime}$ for $P$ in $\psi$ ).

Proposition 2.1. Assume $|L|<\mu,\left|L^{\dagger}\right|<\mu$ for some infinite $\mu$ and that $L^{\dagger}$ is ( $\mu, \omega)$-compact; then the following are equivalent:

(i) CRAIG $\left(L^{\dagger}, L^{\dot{\eta}}\right)$,

(ii) $\operatorname{ROB}\left(L^{\dagger}, L^{\dagger}\right)$,

(iii) $\mathrm{WROB}\left(L^{\dagger}, L^{\dagger}\right)$.

The proof is left as an exercise.

Let DEF be any of the prefixes CRAIG, BETH, WBETH, ROB, WROB or $\Delta$ Int. The following is immediate: 
Proposition 2.2. If $L^{*}, L^{\dagger}, L^{* *}, L^{+t}$ are logics, $L^{* *}<L^{*}$ and $L^{\dagger}<L^{\dagger+}$, then $\operatorname{DEF}\left(L^{*}, L^{\dagger}\right)$ implies $\operatorname{DEF}\left(L^{* *}, L^{\dagger}\right)$.

THEOREM 2.3. The following diagrams are true:

$$
\begin{gathered}
\operatorname{CRAIG}\left(L^{*}, L^{\dagger}\right) \\
\Delta \operatorname{Int}\left(L^{*}, L^{\dagger}\right) \quad \operatorname{BETH}\left(L^{*}, L^{\dagger}\right) \\
\text { WBETH}\left(L^{*}, L^{\dagger}\right) \\
\operatorname{CRAIG}\left(L^{*}, L^{\dagger}\right) \quad \operatorname{ROB}\left(L^{*}, L^{\dagger}\right) \\
\text { WROB }\left(L^{*}, L^{\dagger}\right)
\end{gathered}
$$

The proofs are left as an exercise.

Before we can discuss other implications we need some facts from [MSS]:

Every logic $L^{*}$ has a least extension $\Delta\left(L^{*}\right)$ which satisfies

$$
\Delta \operatorname{Int}\left(\Delta\left(L^{*}\right), \Delta\left(L^{*}\right)\right) \text {. }
$$

Similarly for $\operatorname{BETH}\left(B\left(L^{*}\right), B\left(L^{*}\right)\right)$ and $\mathrm{WBETH}\left(\mathrm{WB}\left(L^{*}\right), \mathrm{WB}\left(L^{*}\right)\right)$. The following is taken from [MSS].

THEOREM 2.4. Assume that $L^{*}$ is a logic.

(i) If $L^{*}$ is $(\kappa, \lambda)$-compact, so are $\mathrm{WB}\left(L^{*}\right)$ and $\Delta\left(L^{*}\right)$.

(ii) If $L^{*}$ satisfies $\mathrm{LS}_{\mu}(\kappa, \lambda)$ so do $\Delta\left(L^{*}\right)$ and $\mathrm{WB}\left(L^{*}\right)$.

(iii) $\mathrm{WB}\left(L^{*}\right)$ does not preserve the Karp-property (neither do $B\left(L^{*}\right)$ nor $\left.\Delta\left(L^{*}\right)\right)$.

It is an open question, whether a similar theorem holds for $B\left(L^{*}\right)$. There are many ways to define extensions $L^{\dagger}$ (minimal extensions) of a given logic $L^{*}$ which satisfy CRAIG $\left(L^{\dagger}, L^{\dagger}\right)$. The problem is to define one which is in some reasonable sense canonical and satisfies an analogue of Theorem 2.4.

THEOREM 2.5. (i) $\operatorname{BETH}\left(L^{*}, L^{\dagger}\right) \nRightarrow \Delta \operatorname{Int}\left(L^{*}, L^{\dagger}\right)$.

(ii) $\Delta \operatorname{Int}\left(L^{*}, L^{\dagger}\right) \nRightarrow \operatorname{BETH}\left(L^{*}, L^{\dagger}\right)$.

So no other implication in the diagram of 2.2 does hold.

Proof. To prove 2.5 we shall construct two new logics and calculate their BETH-closure and $\Delta$-closure:

(i) Let $L^{\text {wo }}=L_{\omega w}\left[Q^{\text {wo }}\right]$ be the logic obtained from $L_{\omega w}$ by adjunction of a binary quantifier $Q^{\mathrm{wo}} x y \varphi(x, y)$ which says that $\varphi(x, y)$ well-orders its field. $L^{\text {wo }}$ satisfies $\mathrm{LS}(\omega)$ since it is a special case of a securable quantifier discussed in [Ma1]. Note that by Theorem 6.1 below WBETH $\left(L^{\text {wo }}, L^{\text {wo }}\right)$ does not hold. To calculate $B\left(L^{\text {wo }}\right)$ we prove:

LEMMA 2.6. If $L$ contains only unary predicate symbols and equality, then $L_{\text {ww }}=L^{\text {wo }}=B\left(L^{\text {wo }}\right)$. 
OUTLINE OF PROOF. We prove by induction on the explicit construction of $B\left(L^{\text {wo }}\right)$ that for such $L$ as in the hypothesis there are no formulas satisfying the hypothesis of the Beth-property in the nontrivial way.

To calculate $\Delta\left(L^{\mathrm{wo}}\right)$ we observe:

LEMMA 2.7. $L\left(Q_{0}\right) \subseteq \Delta\left(L^{\mathrm{wo}}\right)$.

Proof. Since $\langle\omega,<\rangle$ is characterizable by a sentence of $L^{\text {wo }}$ the class of finite sets is in $\operatorname{PC}\left(L^{\mathrm{wo}}\right)$, as well as the class of infinite sets.

Now Lemma 2.6 shows us that $L\left(Q_{0}\right)$ is not a sublogic of $B\left(L^{\text {wo }}\right)$, but by 2.7 it is a sublogic of $\Delta\left(L^{\mathrm{wo}}\right)$, hence of $\Delta\left(B\left(L^{\mathrm{wo}}\right)\right) \neq B\left(L^{\mathrm{wo}}\right)$.

(ii) In [MS3] it is proven that $\Delta\left(L_{\omega \omega}\left[Q_{1}\right]\right)$ does not satisfy Beth's theorem.

THEOREM 2.8. (i) CRAIG $\left(L^{*}, L^{\dagger}\right) \Rightarrow \operatorname{ROB}\left(L^{*}, L^{\dagger}\right)$,

(ii) $\operatorname{ROB}\left(L^{*}, L^{\dagger}\right) \nRightarrow \mathrm{WBETH}\left(L^{*}, L^{\dagger}\right)$,

(iii) $\Delta \operatorname{Int}\left(L^{*}, L^{\dagger}\right) \Rightarrow \mathrm{WROB}\left(L^{*}, L^{\dagger}\right)$.

Proof. (i) Put $L^{*}=L_{\omega, \omega}, L^{\dagger}=L_{\omega, \omega}$. We have CRAIG $\left(L_{\omega, \omega}, L_{\omega, \omega}\right)$ by [Ke2, p. 19] and not $\operatorname{ROB}\left(L_{\omega_{1} \omega}, L_{\omega_{1} \omega}\right)$ by [Ke2, exercise 4, p. 22] (cf. also 2.14).

(ii) Put

$$
L^{*}=L_{\omega_{1} \omega_{1}} \text { and } L^{\dagger}=L_{\infty \infty}
$$

Malitz showed that WBETH( $\left.L_{\omega_{1} \omega_{1}}, L_{\infty}\right)$ fails (cf. [GH, Theorem 2]). To prove $\operatorname{ROB}\left(L_{\omega_{1} \omega_{1}}, L_{\infty \infty}\right)$ we note first that a complete theory in $L_{\infty \infty}$ is always categorical, so the Robinson-property follows trivially.

(iii) Hutchinson (cf. [MSS]) showed that $\Delta\left(L_{\omega \omega}\left(Q_{1}\right)\right)$ (with quantifier "there exist uncountably many") does not satisfy Craig's theorem, in fact not $\operatorname{CRAIG}\left(L_{\omega \omega}(Q), \Delta\left(L_{\omega \omega}(Q)\right)\right)$. But $\Delta\left(L_{\omega \omega}(Q)\right)$ is $(\omega, \omega)$-compact, so by Propositions 2.1 and 2.2 the result follows, with $L^{*}=L^{\dagger}=\Delta\left(L_{\omega \omega}(Q)\right)$.

THEOREM 2.9. (i) $\operatorname{BETH}\left(L^{*}, L^{\dagger}\right) \nRightarrow \mathrm{WROB}\left(L^{*}, L^{\dagger}\right)$.

(ii) All possible relations between two definability properties are exhibited.

Proof. (i) Let $L^{\text {cf } 1}$ be the logic obtained from $L_{\omega \omega}$ by adjunction of a binary quantifier $Q^{\text {cf }{ }^{1}} x y \varphi(x, y)$ which says that $\varphi(x, y)$ orders its field in an $\omega_{1}$-like way. As in 2.4(i) we need a lemma.

LEMMA 2.10. If $L$ contains only unary predicate symbols and equality then $B\left(L^{\text {cf } 1}\right)=L^{\text {cf } 1}=L_{\omega \omega}$.

The proof is as for Lemma 2.6. To end the proof of 2.9 we need:

Proposition 2.11. $L^{\text {cf } 1}$ does not satisfy $\operatorname{WROB}\left(L^{\text {cf } 1}, L^{\text {cf } 1}\right)$. 
Proof. By Lemma 2.10 and first order model theory the $L$-theory $T_{\text {inf }}$ of infinite sets for $L=\{=\}$ is complete in $L^{\text {cf } 1}$. Let $\varphi_{1}$ say that an ordering $<_{1}$ of a model is $\omega_{1}$-like and let $\varphi_{2}$ say that an ordering $<_{2}$ is a proper initial segment of an $\omega_{1}$-like ordering (using additional predicates). Clearly $T_{\text {inf }} \cup$ $\left\{\varphi_{i}\right\}$ has a model for each $i=1,2$, but $T_{\text {inf }} \cup\left\{\varphi_{1}, \varphi_{2}\right\}$ has no model.

To prove (ii) of 2.9 one uses a finitary checking argument.

For the rest of this section we look at Karp-logics, modifying results of Barwise.

TheOREM 2.12. Let $L^{*}$ be a Karp-logic (hence $L^{*}<_{\mathrm{Th}} L_{\infty \omega}$ ).

(i) $\operatorname{BETH}\left(L^{*}, L_{\infty \omega}\right)$ implies $\operatorname{WROB}\left(L^{*}, L_{\infty \omega}\right)$.

(ii) $\mathrm{WROB}\left(L^{*}, L_{\infty \omega}\right)$ implies that $L^{*}$ satisfies $\mathrm{LS}(\omega)$.

COROLLARY 2.13 [Ba]. If $L^{*}$ is a Karp-logic and CRAIG $\left(L^{*}, L^{*}\right)$ holds then LS( $\omega)$ holds.

The corollary follows from the theorem via Proposition 2.2.

Proof of THE THEOREM. (i) will follow from Theorem 5.3 taking $L^{*}=L^{\dagger}$ $=L_{\infty \omega}$.

(ii) By 1.2 and 1.5 we may assume that $\langle\omega,\langle\rangle$ is characterizable by $\varphi \in L^{*}$. Assume for contradiction that $\psi$ is a formula in $L^{*}$ with no countable but some uncountable models. Clearly $\{\varphi, \psi\}$ has no model. Let $T$ be the $L_{\infty}$-theory of infinite sets. Since all infinite sets are partially isomorphic, $T$ is $L_{\infty \omega}$-complete. But $T \cup\{\varphi\}$ and $T \cup\{\psi\}$ have models, a contradiction.

Theorem 2.14. Assume $L^{*}$ is a Karp-logic, $\operatorname{ROB}\left(L^{*}, L^{*}\right)$ holds and for every L-structure $\mathfrak{M}, \mathrm{Th}_{L}^{*}(\mathfrak{M})$ is equivalent to a set. Then $L^{*}=L_{\omega \omega}$.

Proof. Again we can assume that $\langle\omega,\langle\rangle$ is characterizable by a sentence $\varphi$ in $L^{*}$. Put $\mathfrak{R}_{1}=\left\langle\omega,\langle, \ldots\rangle\right.$ and $\mathfrak{M}_{2}=\left\langle C, c_{\alpha}: \alpha\left\langle\omega_{1}\right\rangle\right.$, a pure set with equality and $\omega_{1}$-many distinct constants.

Put $\mathfrak{M}_{3}^{0}=\left|\omega_{0}\right|$ and $\mathfrak{M}_{3}^{1}=\left|\omega_{1}\right|$. Using the Back and Forth characterization of $\simeq_{p}$ (cf. [Ba1]) we get that $\left[\mathfrak{M}_{1}, \mathfrak{M}_{2}, \mathfrak{M}_{3}^{0}\right] \simeq_{p}\left[\mathfrak{R}_{1}, \mathfrak{M}_{2}, \mathfrak{M}_{3}^{1}\right]$. Put

$$
T=\mathrm{Th}_{L_{1}}\left[\mathfrak{M}_{1}, \mathfrak{M}_{2}, \mathfrak{M}_{3}^{0}\right]=\operatorname{Th}_{L_{1}}\left[\mathfrak{M}_{1}, \mathfrak{M}_{2}, \mathfrak{M}_{3}^{1}\right],
$$

so $T$ can be assumed to be a set and $T$ is complete. Let $\psi_{i}$ say that $g_{i}$ is a bijection from $\left|\mathfrak{M}_{i}\right|$ to $\left|\mathfrak{M}_{3}\right|(i=1,2)$. Clearly $T \cup\left\{\psi_{i}\right\}$ has a model $(i=1,2)$ but $T \cup\left\{\psi_{1}, \psi_{2}\right\}$ has no model since $\mathfrak{R}_{1} \vDash \varphi$ and therefore $\mathfrak{M}_{1}$ is countable and $\mathfrak{M}_{2}$ is uncountable, a contradiction.

Corollary 2.15. Assume $L^{*} \leqslant L_{\infty \omega}$ and $\operatorname{ROB}\left(L^{*}, L_{\infty \omega \omega}\right)$. Then $L^{*}=L_{\omega \omega}$.

Proof. Note that for every structure $M, \operatorname{Th}_{L_{\infty}}(M)$ is equivalent to a set, and repeat the proof of 2.14 . 
3. Feferman-Vaught-type theorems. One basis construction in abstract model theory is the operation of taking sums of given models. In its simplest form we have the operation of pairing which associates with two structures $\mathfrak{A}, \mathfrak{B}$ the structure $[\mathfrak{A}, \mathfrak{B}]$ for the language $L$ which is the many-sorted disjoint union of the languages of $\mathfrak{A}$ and $\mathfrak{B}$, respectively. Similarly one can form triplets, etc., or more complicated objects, of which one will be studied in $\$ 5$.

Definition. Let $L_{1}, L_{2}$ be languages and $L_{i}^{*}, L_{i}^{\dagger}(i=1,2)$ logics with $L_{i}^{*}<L_{i}^{\dagger}$. Let $\mathfrak{A}_{i}$, $\mathfrak{A}_{i}^{\prime}$ be $L_{i}$-structures. We say that $\mathrm{FV}_{p}\left(L^{*}, L^{\dagger}\right)$ holds if, whenever $\mathfrak{A}_{i} \equiv \mathfrak{U}_{i}^{\prime}\left(L_{i}^{\dagger}\right)$ for $i=1,2$ and $L_{3}$ is the language of the pair $\left[\mathfrak{A}_{00}, \mathfrak{A}_{1}\right]$ then $\left[\mathfrak{A}_{1}, \mathfrak{A}_{2}\right] \equiv\left[\mathfrak{U}_{1}^{\prime}, \mathfrak{X}_{2}^{\prime}\right]\left(L_{3}^{*}\right)$. We say that $\operatorname{WFV}_{p}\left(L^{*}, L^{\dagger}\right)$ holds whenever $\mathrm{FV}_{p}\left(L^{*}, L^{\dagger}\right)$ holds for $L_{1}=L_{2}=L$ and $\mathfrak{U}_{1} \equiv \mathfrak{A}_{2}\left(L^{\dagger}\right)$ (F stands for Feferman, $V$ for Vaught and $W$ for Weak).

Remember that the pair $\left[\mathfrak{U}_{1}, \mathfrak{A}_{2}\right]$ is the structure with the universes from $\mathfrak{X}_{1}$ and from $\mathfrak{A}_{2}$ and relations acting on the appropriate universes. It is, loosely speaking, the disjoint union of the structures $\mathfrak{X}_{1}$ and $\mathfrak{X}_{2}$ with variables ranging over the universes of $\mathfrak{X}_{1}$ and variables ranging over the universes of $\mathfrak{X}_{2}$.

Note that if $L^{* *}<L^{*}$ and $L^{\dagger+}>L^{\dagger}$, then $X\left(L^{*}, L^{\dagger}\right)$ implies $X\left(L^{* *}, L^{+}\right)$ where $X$ is one of the properties above.

TheOREM 3.1. Assume $L^{*}$ is a logic which satisfies WBETH $\left(L^{*}, L^{*}\right)$ and $\mathrm{WFV}_{p}\left(L^{*}, L^{*}\right)$ and $\mathrm{LS}(\lambda)$ for some $\lambda<\beth_{\omega_{1} c k}$. Then $\langle\omega,<\rangle$ is not characterizable by a single sentence.

Proof. We give a proof for the case that $\lambda<\beth_{\omega}$, the general case being essentially the same but more complicated. Assume for contradiction that $\varphi \in L^{*}$ does characterize $\langle\omega,\langle\rangle$. By assumption and 1.6 there are at most $2^{2^{2^{4}}}$-many theories over countable types. Consider the structure $\mathfrak{A}=$ $\left\langle V, \omega, P^{n}(\omega), \varepsilon\right\rangle$ where $V=\cup_{k \in \omega} P^{k}(\omega), n \in \omega$, such that $2^{2^{2^{4}}}<z_{n}$, and $P^{n}(\omega)$ is the $n$th iteration of the power set operation. Now consider the structure $[\mathfrak{A}, \mathfrak{X}]$ and let $\psi$ be the formula which says " $\omega$ is standard" using $\varphi$ :

" $F$ is a partial map from $V$ of the first sort (i.e., $V_{1}$ ) to $V$ of the second sort (i.e., $V_{2}$ )";

" $F$ and $F^{-1}$ preserve $\varepsilon$ ";

" $F$ is hereditary, i.e. if $F$ is defined on $x$ and $y \in x$, it is defined on $y$ ";

"The domain of $F$ is maximal".

Clearly $\psi$ defines $F$ implicitly and $\psi$ is in $L^{*}$. Since there are at most $2^{2^{2^{\lambda}}}$-many theories we may find two structures $\mathfrak{X}_{1}=\left\langle V_{1}, \omega, P_{1}, \varepsilon\right\rangle$ and $\mathfrak{X}_{2}=$ $\left\langle V_{2}, \omega, P_{2}, \varepsilon\right\rangle$ such that $\mathfrak{X}_{1} \equiv \mathfrak{X}_{2}\left(L^{*}\right)$ and $P_{1} \neq P_{2}$ but $P_{1}, P_{2} \subset P^{n}(\omega)$.

So by $\operatorname{WFV}_{P}\left(L^{*}, L^{*}\right)$ we have $\left[\mathfrak{A}_{1}, \mathfrak{A}_{2}\right] \equiv\left[\mathfrak{I}_{1}, \mathfrak{U}_{1}\right]\left(L^{*}\right)$. But assuming that $\theta(x, y)$ defines $F$ explicitly in $L^{*}$ we get that $\theta(x, y)$ defines a map with domain $P_{1}$ on $\left[\mathfrak{A}_{1}, \mathfrak{X}_{1}\right]$, but not on $\left[\mathfrak{X}_{1}, \mathfrak{X}_{2}\right]$, a contradiction. So $\langle\omega,<\rangle$ is not characterizable in $L$. In fact with more coding it suffices to assume that $\lambda<\beth_{\omega_{1} c k}$. 
COROLlaRY 3.2. The following properties characterize $L_{\omega \omega}$ :

(i) $\operatorname{LS}(\omega), \operatorname{WFV}_{P}\left(L_{\omega \omega}, L_{\omega \omega}\right)$ and $\operatorname{WBETH}\left(L_{\omega \omega}, L_{\omega \omega}\right)$.

(ii) The Karp-property, $\operatorname{WFV}_{P}\left(L_{\omega \omega}, L_{\omega \omega}\right)$ and $\operatorname{WBETH}\left(L_{\omega \omega}, L_{\omega \omega}\right)$.

Compare 3.2 also with 4.2.

The following is an abstract version of a result of Malitz [MI] cf. also [GH].

THEOREM 3.3. Let $L^{*}$ be a set for all languages $L$ and assume that $L^{\square} \subset L^{\dagger} \subset L^{*}$ are three logics such that $\operatorname{WBETH}\left(L^{\square}, L^{\dagger}\right)$ and $\mathrm{WFV}_{P}\left(L^{\dagger}, L^{*}\right)$. Then the class of well-orderings is not $\mathrm{EC}\left(L^{\square}\right)$.

Proof. Let $\alpha\left\langle\beta\right.$ be two infinite ordinals such that $\langle\alpha, \varepsilon\rangle \equiv\langle\beta, \varepsilon\rangle\left(L^{*}\right)$. They exist for $L^{*}$ is a set. Now look at two-sorted structures of the form $[\mathfrak{A}, \mathfrak{B}]$ where $\mathfrak{A}$ and $\mathfrak{B}$ are well-orderings and let $F$ be a binary function symbol with domain $\mathfrak{A}$ and range $\mathfrak{B}$. Assume for contradiction that the class of well-orderings is $\operatorname{EC}(L)$.Let $\theta$ be a sentence expressing that:

(i) $\mathfrak{A}$ and $\mathfrak{B}$ are well-orderings,

(ii) $F$ is an order-preserving map which is one-to-one,

(iii) either $F$ is an embedding of $\mathfrak{U}$ into $\mathfrak{B}$ or of $\mathfrak{B}$ into $\mathfrak{A}$.

Now $\theta$ satisfies the hypothesis of $\operatorname{WBETH}\left(L^{\square}, L^{t}\right)$. Therefore there is $\varphi(x, y)$ in $L^{\dagger}, L$ the language of $[\mathfrak{A}, \mathfrak{B}]$ without $F$, defining $F$. Now put $\mathfrak{A}=\langle\alpha, \varepsilon\rangle, \mathfrak{B}=\langle\beta, \varepsilon\rangle . \operatorname{By~WFV~}_{P}\left(L^{\dagger}, L^{*}\right)$ we have $[\mathfrak{A}, \mathfrak{B}] \equiv[\mathfrak{O}, \mathfrak{U}]\left(L^{\dagger}\right)$. Put $\varphi_{1}$ to express that $\varphi(x, y)$ is an embedding of $\mathfrak{A}$ into $\mathscr{B}$ and $\varphi_{2}$ that $\varphi(x, y)$ is an embedding of $\mathfrak{B}$ into $\mathfrak{A}$.

So we have $[\mathfrak{U}, \mathfrak{B}] \vDash \varphi_{1} \wedge \neg \varphi_{2}$ and $[\mathfrak{B}, \mathfrak{U}] \vDash \varphi_{2} \wedge \neg \varphi_{1}$, a contradiction.

Theorem 3.1 can be easily generalized to:

THEOREM 3.4. Let $L^{*} \subset L^{\dagger} \subset L^{\square}$ be three logics, such that $\operatorname{WBETH}\left(L^{*}, L^{\dagger}\right)$ and $\mathrm{WFV}_{P}\left(L^{\dagger}, L^{\dagger}\right)$ and $L$ satisfies $\operatorname{LS}(\lambda)$ for some $\lambda<z_{\omega_{1}}$. Then $\left\langle\omega,\langle\rangle\right.$ is not characterizable by a single sentence of $L^{*}$.

In many cases, where $\mathrm{FV}_{P}$ does not hold, it may still hold for specially chosen structures. How this is used is shown in [MS2], [MS3].

4. Uniform reduction. In Feferman [Fe2] and Makowsky [Ma2] a property of logics is studied which has two advantages: It comes nearer to the original Feferman-Vaught theorem and it is directly related to Craig's theorem.

Let $\mathfrak{A}_{i}$ be an $L_{i}$-structure $(i=1,2)$ and let $L_{3}$ be a language for $\left[\mathfrak{A}_{1}, \mathfrak{A}_{2}\right]=$ $P\left(\mathfrak{A}_{1}, \mathfrak{A}_{2}\right)$. For a logic $L^{*}$ we say that $\mathrm{UR}_{P}\left(L^{*}\right)$ holds if for every sentence $\varphi$ of $L_{3}^{*}$ there exists a pair of sequences of formulae $\psi_{1}^{1}, \ldots, \psi_{n_{1}}^{1}$ of $L_{1}^{*}$ and $\psi_{1}^{2}, \ldots, \psi_{n_{2}}^{2}$ of $L_{2}^{*}$ and a Boolean function $B \in 2^{n_{1}+n_{2}}$ such that

$$
\left[\mathfrak{A}_{1}, \mathfrak{A}_{2}\right] \vDash \varphi \text { iff } B\left(a_{1}^{1}, \ldots, a_{n_{1}}^{1}, a_{1}^{2}, \ldots, a_{n_{2}}^{2}\right)=1
$$


where

$$
a_{j}^{i}= \begin{cases}0 & \text { if } \mathscr{U}_{i} \vDash \neg \psi_{j}^{i}, \\ 1 & \text { if } \mathscr{U}_{i} \vDash \psi_{j}^{i} .\end{cases}
$$

THEOREM 4.1. Assume $\mathrm{UR}_{P}\left(L^{*}\right)$ holds for a logic $L^{*}$. Then $\mathrm{FV}_{P}\left(L^{*}, L^{*}\right)$ holds.

The proof is left to the reader. We now want to generalize $\mathrm{UR}_{P}$ (uniform reduction) to a more general class of operations. Let $L_{i}(i=1, \ldots, n \in \omega)$ be languages, $\mathfrak{X}_{i}$ be $L_{i}$-structures and $R$ an $n$-ary relation on structures such that the $i$ th argument is an $L_{i}$-structure. Let $L_{0}$ be the language for a structure $\left[\mathfrak{A}_{1}, \ldots, \mathfrak{A}_{n}\right] . R$ is said to be projective in the logic $L^{*}$ if there is a $P C^{\prime}$-class $K$ for $L^{*}$ such that $R\left(\mathfrak{A}_{1}, \ldots, \mathfrak{X}_{n}\right)$ iff $\left[\mathfrak{A}_{1}, \ldots, \mathfrak{A}_{n}\right] \in K$. A sentence $\varphi \in L_{n}^{*}$ is said to be invariant on the range of $R$ if for all $\mathfrak{X}_{1}, \ldots, \mathfrak{A}_{n-1}, \mathfrak{A}_{n}, \mathfrak{A}_{n}^{\prime}$ with $R\left(\mathfrak{A}_{1}, \mathfrak{U}_{n-1}, \mathfrak{A}_{n}\right)$ and $R\left(\mathfrak{A}_{1}, \ldots, \mathfrak{X}_{n-1}, \mathfrak{X}_{n}^{\prime}\right)$ we have $\mathfrak{X}_{n} \vDash \varphi$ iff $\mathfrak{X}_{n}^{\prime} \vDash \varphi$.

An $n$-tuple of sequences of formulas $\bar{\psi}_{1}, \ldots, \bar{\psi}_{n-1}$ with $\bar{\psi}_{k}=\psi_{1}^{k}, \ldots, \psi_{m k}^{k}$ and $\psi_{i}^{k}$ in $L_{k}^{*}$ together with a Boolean function $B \in 2^{m}, m=\sum_{k=0}^{n-1} m_{k}$ is called an associate pair for $\varphi$ on the domain of $R$ if for all $\mathfrak{A}_{1}, \ldots, \mathfrak{U}_{n-1}, \mathfrak{U}_{n}$ we have that $R\left(\mathfrak{A}_{1}, \ldots, \mathfrak{A}_{n}\right)$ implies $\mathfrak{A}_{n} \vDash \varphi$ iff $B\left(a_{1}^{1}, \ldots, a_{m_{1}}^{1}, a_{1}^{2}, \ldots, a_{m_{n-1}}^{n-1}\right)=1$ where

$$
a_{j}^{i}= \begin{cases}1 & \mathfrak{A}_{i} \vDash \psi_{j}^{i}, \\ 0 & \text { otherwise. }\end{cases}
$$

A logic $L^{*}$ is said to satisfy $\mathrm{UR}_{n}\left(L^{*}\right)$ (uniform reduction for $n$-ary relations) if for every $n$-ary relation $R$ which is projective in $L^{*}$ and for every $\varphi$ in $L_{n}^{*}$ which is invariant on the range of $R$ there is an associate pair for $\varphi$ on the domain of $R$.

THEOREM 4.2. (i) $\mathrm{UR}_{1}\left(L^{*}\right)$ iff $\operatorname{CRAIG}\left(L^{*}, L^{*}\right)$.

(ii) $\mathrm{UR}_{2}\left(L^{*}\right)$ implies $\mathrm{UR}_{P}\left(L^{*}\right)$.

(iii) $\mathrm{UR}_{2}\left(L^{*}\right)$ implies $\mathrm{UR}_{n}\left(L^{*}\right)$.

The proofs of (i)-(iii) may be found in [Fe2] and [Ma1].

COROLlaRY 4.3. If $L^{*}$ is a logic with $\mathrm{UR}_{2}\left(L^{*}\right)$ and the Karp-property and $L^{*}$ is a set, then $L^{*}=L_{\omega \omega}$.

Proof. Use Theorems 4.2 and 2.14.

5. Constructing implicit definitions. In this section we study a construction which enables us to obtain implicit definitions. The construction originates in the ideas of Friedman [Fr] and Gregory [Gr]. Shelah realized that the construction can be formulated in an abstract setting.

Let $L=L_{0} \cup\{P\}$ be a language with a distinguished predicate symbol $P$ 
(unary for simplicity). Let $M=L \cup\{f, C\}$ where $f$ is a unary function symbol and $C$ a constant, both not in $L$. Let $\mathfrak{A}, \mathfrak{B}$ be $L$-structures.

We define now $S_{P}^{i}(\mathfrak{A}, \mathfrak{B})(i=0,1)$.

(i) $S_{P}^{i}(\mathfrak{A}, \mathfrak{B})$ is an $M$-structure with universe $N$ of cardinality card( $\left.\mathfrak{A}\right)+$ $\operatorname{card}\left(\mathfrak{B )}+\aleph_{0}\right.$.

(ii) $f$ is a function on $N$ such that

(a) $f(x)=x$ iff $C=x$,

(b) for all $a \in N$ there is $n \in \omega$ with $f^{n}(a)=C$,

(c) $f$ is onto.

Denote $f^{-1}(a)-\{a\}$ by $N_{a}$ (see Figure 1).

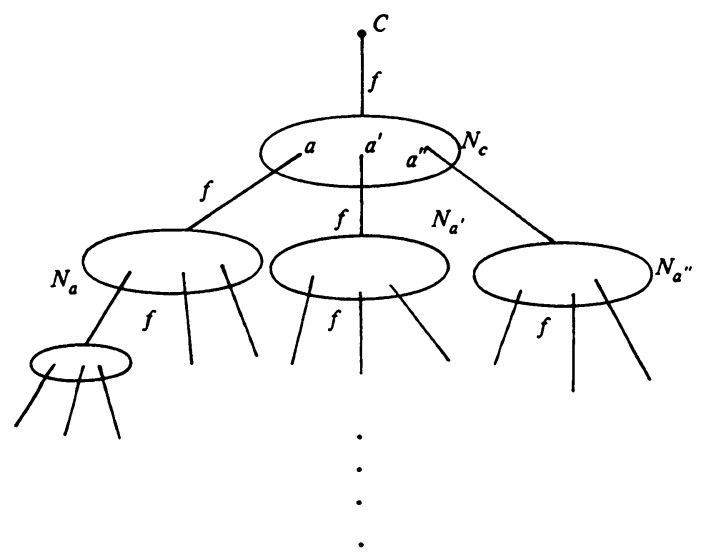

FIGURE 1

(iii) There is a bijection $i_{a}: \mathcal{C} \rightarrow N_{a}(i=0,1)$ where $\mathcal{C}$ is either $\mathfrak{A}$ or $\mathfrak{B}$.

$i_{a}$ makes $N_{a}$ naturally into an $L_{0} \cup\{P\}$-structure which we shall denote also by $\boldsymbol{N}_{a}$.

(iv) for each relation symbol $R \in L$ (or function symbol) put $R_{a}$ to be its interpretation on $N_{a}$ and put $R_{N}=\cup_{a \in N} R_{a}$ its interpretation on $N$.

(v) $P_{N}$ is defined by $a \in P_{N} \Rightarrow N_{a} \cong \mathfrak{A}, a \notin P_{N} \Rightarrow N_{a} \simeq \mathfrak{B}$.

(vi) $C \in P_{N}$ if $i=1, C \notin P_{N}$ if $i=0$.

$S_{P}^{0}(\mathfrak{A}, \mathfrak{B})$ and $S_{P}^{1}(\mathfrak{A}, \mathfrak{B})$ differ only with respect to $N_{C} . S_{P}^{i}(\mathfrak{A}, \mathfrak{B})$ is a tree with root $C$ and nodes $N_{a}$. At the node $N_{a}$ it splits $\left|N_{a}\right|$-many times. Each branch has length $\omega . N_{a} \cong \mathfrak{A}$ iff $f(a) \in P$ and $N_{a} \cong \mathfrak{B}$ iff $f(a) \notin P$ (see Figure 2).

Now we put $T_{P}^{i}(\mathfrak{A}, \mathfrak{B}):=S_{P}^{i}(\mathfrak{A}, \mathfrak{B}) \backslash L_{0} \cup\{f, c\}$. We say that WFVT $\left(L^{*}, L^{\dagger}\right)$ holds if $\mathfrak{A} \equiv \mathfrak{B}\left(L^{\dagger}\right)$ implies that $T_{P}^{0}(\mathfrak{A}, \mathfrak{B}) \equiv T_{P}^{1}(\mathfrak{A}, \mathfrak{B})\left(L^{*}\right)$.

Proposition 5.1. If $\left\langle\omega,\langle\rangle\right.$ is $\operatorname{PC}\left(L^{*}\right)$. Then $\mathrm{UR}_{2}\left(L^{*}\right)$ implies WFVT $\left(L^{*}, L^{*}\right)$. 


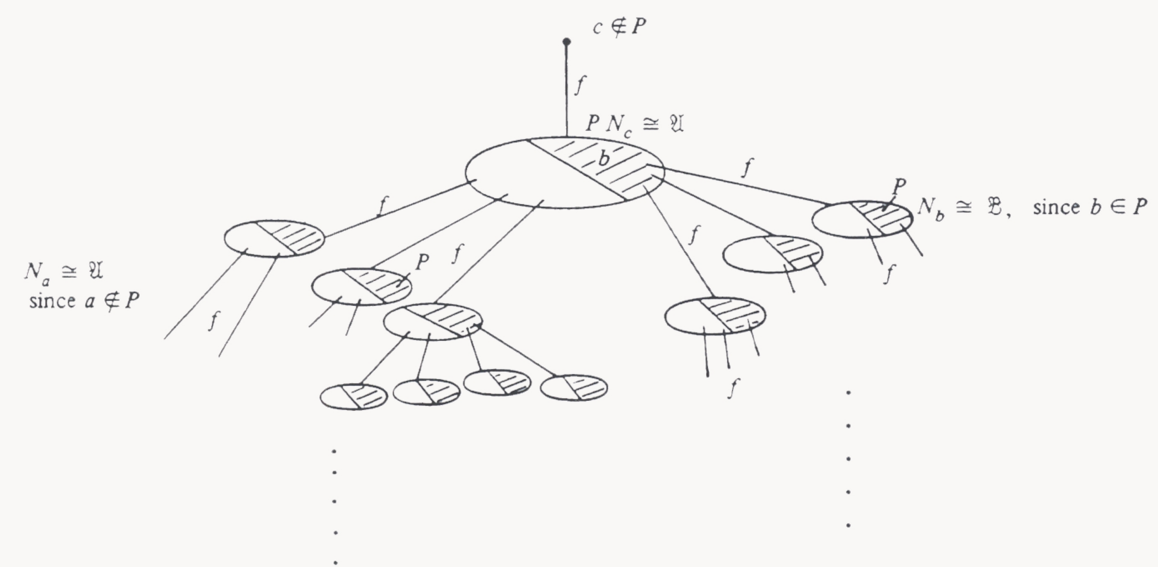

FIGURE 2

Proof. $S_{P}^{i}(\mathfrak{A}, \mathfrak{B})$ is a projective operation via the characterization of $\left\langle\omega,\langle\rangle\right.$, if we replace it by a ternary operation $S_{P}\left(\mathfrak{A}^{\prime}, \mathfrak{A}, \mathfrak{B}\right)$ restricted to the cases $\mathfrak{U}^{\prime} \cong \mathfrak{A}$ or $\mathfrak{U}^{\prime} \cong \mathfrak{B}$, so that the first argument describes $N_{C}$ and the second argument describes $N_{a}$ for $a \in P$, the third $N_{a}$ for $a \notin P$.

LEMMA 5.2. Let $L^{*}$ be a logic, $L$ a language and $P_{i}(i=0,1,2)$ be unary predicate symbols not in $L$.

Let $\varphi_{i}(i=1,2)$ be sentences having a model but $\left\{\varphi_{1}, \varphi_{2}\right\}$ has no model. Then there is a sentence $\psi \in\left(L \cup\left\{f, c, P_{0}\right\}\right)^{*}(f, c$ not in $L)$ such that:

(i) Every $L \cup\{f, c\}$-structure $\mathfrak{A}$ has at most one expansion $\mathfrak{X}^{*} \vDash \psi$.

(ii) $S_{P_{0}}^{i}(\mathfrak{A}, \mathfrak{B}) \vDash \psi$ provided $\mathfrak{A} \vDash \varphi_{1}$ and $\mathfrak{B} \vDash \varphi_{2}$ where $P_{1}$ and $P_{2}$, respectively, are substituted for $P_{0}$.

Proof. (i) Let $\psi$ be $\psi_{1} \wedge \psi_{2}$ with

$$
\begin{gathered}
\psi_{1}: f(c)=c \wedge f(x)=x \Leftrightarrow x=C \wedge \text { " } f \text { is onto"; } \\
\psi_{2}: a \in P_{0} \Rightarrow N_{a}=\left\langle f^{-1}(a)-\{a\}, L \cup\left\{f, c, P_{0}\right\}\right\rangle \vDash \varphi_{1}, \\
a \notin P_{0} \Rightarrow N_{a}=\left\langle f^{-1}(a)-\{a\}, L \cup\left\{f, c, P_{0}\right\}\right\rangle \vDash \varphi_{2} .
\end{gathered}
$$

Note that $\psi_{1} \in L_{\omega \omega}$ and $\psi_{2} \in L^{*}$. Now let $\mathfrak{A}$ be an $L \cup\{f, c\}$-structure and $\left\langle\mathfrak{U}, P_{i}\right\rangle \vDash \psi(i=1,2)$ (ignoring substitutions of $P_{i}$ for $P_{0}$ in $\left.\psi\right)$. We have to show that $P_{1}=P_{2}$. If $a \in P_{1}$ then $N_{a} \vDash \varphi_{1}$, if $a \notin P_{2}$ then $N_{a} \vDash \varphi_{2}$ so

$$
\left\langle f^{-1}(a)-\{a\}, L \cup\left\{f, c, P_{1}, P_{2}\right\}\right\rangle \vDash \varphi_{1} \wedge \varphi_{2},
$$

a contradiction.

(ii) is obvious by the construction of $S_{P}^{i}(\mathfrak{A}, \mathfrak{B})$.

THEOREM 5.3. Let $L^{*}<L^{\dagger}$ be logics $\varphi_{i}(i=1,2)$ as in Lemma 5.2 and assume that there are $\mathfrak{U}_{i} \vDash \varphi_{i}$ such that $S_{P}^{0}\left(\mathfrak{A}_{0}, \mathfrak{A}_{1}\right) \equiv S_{P}^{1}\left(\mathfrak{U}_{0}, \mathfrak{A}_{1}\right)\left(L^{\dagger}\right)$. Then $\operatorname{BETH}\left(L^{*}, L^{\dagger}\right)$ fails. 
Proof. By Lemma 5.2, $\psi$ defines $\boldsymbol{P}$ implicitly. So assume for contradiction there is $\theta$ in $L^{\dagger}$, an $L \cup\{f, c\}$-formula, such that, for all $L \cup\{f, c\}$ structures $\mathfrak{A}, \mathfrak{U} \vDash \theta(a)$ iff there is an expansion $\mathfrak{U}^{*} \vDash \psi \wedge P(a)$.

Then we have $S_{P}^{0}\left(\mathfrak{A}_{0}, \mathfrak{X}_{1}\right) \vDash \neg \theta(c)$ and $S_{P}^{1}\left(\mathfrak{A}_{0}, \mathfrak{A}_{1}\right) \vDash \theta(c)$ which contradicts $S_{P}^{0}\left(\mathfrak{X}_{0}, \mathfrak{X}_{1}\right) \equiv S_{P}^{1}\left(\mathfrak{A}_{0}, \mathfrak{X}_{1}\right)\left(L^{\dagger}\right)$.

The abstract version of 5.3 is the following:

TheOREM 5.4. Assume $L^{0}<L^{*}<L^{\dagger}$ are logics such that

(i) $\operatorname{BETH}\left(L^{0}, L^{*}\right)$,

(ii) WFVT $\left(L^{*}, L^{\dagger}\right)$.

Then WROB $\left(L^{0}, L^{\dagger}\right)$ holds.

Proof. Assume WROB $\left(L^{0}, L^{\dagger}\right)$ does not hold then we have $\varphi_{i}(i=0,1)$ as in Lemma 5.2 (if the $P_{i}$ are not unary we have the obvious modification of the definition of $S_{P}^{i}(\mathfrak{X}, \mathfrak{B})$ ). Also there are $\mathfrak{A}_{i} \vDash \varphi_{i}$ such that $\mathfrak{X}_{0} \equiv \mathfrak{U}_{1}\left(L^{\dagger}\right)$ and $S_{P}^{0}\left(\mathfrak{A}_{0}, \mathfrak{B}_{1}\right) \equiv S_{P}^{1}\left(\mathfrak{A}_{0}, \mathfrak{B}_{1}\right)\left(L^{*}\right)$, so we can apply Theorem 5.3 and $\operatorname{BETH}\left(L^{0}, L^{*}\right)$ fails.

Note that we have $\operatorname{BETH}\left(L_{\omega_{1} \omega}, L_{\infty \omega}\right)$ and WFVT $\left(L_{\infty \omega,}, L_{\infty \omega}\right)$ but not $\operatorname{ROB}\left(L_{\omega_{1} \omega}, L_{\infty \omega}\right)$ (by 2.15), so the theorem cannot be improved (cf. §8).

6. Compactness and definability. The aim of this section is to find sufficient conditions for a logic to be compact, more precisely to study definability criteria which imply compactness. It was Lindström who observed that:

THEOREM 6.1. If $L^{*}=L_{\omega \omega}\left[Q^{i}\right]_{i<n<\omega}$ is finitely generated and $\operatorname{WBETH}\left(L^{*}, L^{*}\right)$ holds, then $\langle\omega,<\rangle$ is not characterizable by a single sentence.

This implies that $L^{*}$ is recursively compact, i.e. if $\Sigma$ is a recursive set of sentences, such that every finite subset of $\Sigma$ has a model, then $\Sigma$ has a model.

Proof of 6.1. Assume for contradiction that $\psi \in L^{*}$ characterizes $\langle\omega$, $\langle, \bar{R}\rangle$ with $L=\left\{\left\langle, R_{1}, \ldots, R_{m}\right\}\right.$ including enough number theory. Let $L_{1}=L \cup\{c\}$ and $\left\{\psi_{n} \mid n \in \omega\right\}$ a recursive enumeration of $L_{1}$ where $c$ is a constant-symbol. (Here we use the fact that $L^{*}$ is finitely generated.)

Consider the set of formulas of $L_{2}^{*}\left(L_{2}=L_{1} \cup\{P\}\right): \Sigma=\left\{P(n) \Leftrightarrow \psi_{n} \wedge c\right.$ $=n \mid n \in \omega\}$ where $P$ is an unary predicate symbol not in $L_{1} . \Sigma$ is recursive; therefore it is replaceable by some formula $\Phi(P, n)$ using a trick due to Kleene which has been generalized by Lindström for finitely generated logics ([Kl], [MSS]). Now $\Phi \wedge \psi$ defines $P$ implicitly, since the only model of $\Sigma$ is $\langle\omega,\langle, \bar{R}\rangle$ and we have enough number theory.

By assumption there is $\theta\left(c^{\prime}\right) \in L_{1}^{*}$ such that for every $L_{2}$-structure $\mathfrak{A}$ we have $\mathfrak{A} \vDash P(c) \Leftrightarrow \theta\left(c^{\prime}\right)$. But $\neg \theta\left(c^{\prime}\right)$ is $\psi_{m}$ for some $m \in \omega$. So we have $\mathfrak{X} \vDash P(m)$ iff $\mathfrak{A} \vDash \theta(m)$ iff $\mathfrak{A} \vDash \neg \psi_{m}(m)$ iff $\mathfrak{A} \vDash \neg P(m)$, a contradiction.

Väänänen has observed that this can be generalized to an extended version 
of $\operatorname{BETH}\left(L^{*}, L^{*}\right)$ where sentences are replaced by countable sets of sentences in the hypotheses only. The conclusion of 6.1 then changes to $L^{*}$ is $(\omega, \omega)$-compact.

The main result of this section is:

TheOREM 6.2. Assume $L^{*}=L_{\omega \omega}\left[Q^{i}\right]_{i \in \omega}$ and $2^{k_{0}}<2^{*_{n}}$ for some $n \in \omega$. If $L^{*}$ satisfies $\mathrm{ROB}\left(L^{*}, L^{*}\right)$ and $\mathrm{FV}_{P}\left(L^{*}, L^{*}\right)$ then $L^{*}$ is $(\kappa, \omega)$-compact for every $\kappa$.

Theorem 6.2 is due to Shelah and we shall see in Theorem 6.8 and following that the precise set-theoretic conditions. Here the hypothesis that $L^{*}$ is countably generated is for simplicity only. The proof of 6.2 involves two aspects: a set-theoretic one and a model-theoretic one. Instead of proving compactness we study a related notion.

Defintion. $L^{*}$ is $\mu$-rc ( $\mu$-relatively compact) if for any two sets of sentences $\Sigma, \Sigma_{1} \in L^{*}$ with $|\Sigma|=\mu$ and $\left|\Sigma_{1}\right|$ arbitrary, if for every $\Sigma_{0} \subset \Sigma$ with $\left|\Sigma_{0}\right|<\mu, \Sigma_{0} \cup \Sigma_{1}$ has a model then $\Sigma \cup \Sigma_{1}$ has a model.

The following collects some simple facts about $\mu$-rc logics.

LEMMA 6.3. (i) If $\mu$ is regular, $L^{*}$ is $\mu-r c$ and $T_{i}(i<\mu)$ an increasing family of theories of $L^{*}$ such that each $T_{i}$ has a model then $\cup_{i<\mu} T_{i}=T$ has a model.

(ii) If $L^{*}$ is (cf $\mu$ )-rc then $L^{*}$ is $\mu$-rc.

(iii) If $L^{*}$ is $\mu$-rc then $L^{*}$ is $(\mu, \mu)$-compact.

(iv) $L^{*}$ is $(\lambda, \mu)$-compact iff for all $\kappa$ with $\lambda>\kappa>\mu L^{*}$ is $(\kappa, \kappa)$-compact.

(v) If $L^{*}$ is $\kappa$-rc for all $\kappa \lambda \geqslant \kappa \geqslant \mu$ then $L^{*}$ is $(\lambda, \mu)$-compact.

Proof. (i) Let $P_{i}(i<\mu)$ be unary predicates not in $T$ and $\psi_{i}$ be $\exists x P_{i}(x)$. Put $\Sigma_{1}=\left\{\psi_{i} \rightarrow \varphi \mid i<\mu\right.$ and $\left.\varphi \in T_{i}\right\}$ and $\Sigma=\left\{\psi_{i} \mid i<\mu\right\}$. Since $L^{*}$ is $\mu$-rc we verify that $\Sigma$ and $\Sigma_{1}$ satisfy the hypothesis of $\mu$-rc. $|\Sigma|=\mu$ by definition of $\Sigma$. Now let $\Sigma_{0} \subset \Sigma\left|\Sigma_{0}\right|<\mu$. W.1.o.g. $\Sigma$ is well-ordered of order type $\mu$ and $\Sigma_{0}$ is an initial segment say $\Sigma_{0}=\Sigma \uparrow_{\lambda}$. We have to show that $\Sigma_{0} \cup \Sigma_{1}$ has a model. Let $M$ be a model of $T_{\lambda}$ and expand to $M^{\prime}$ such that $P_{i}^{M} \neq \varnothing$ for $i<\lambda$. Obviously $M^{\prime} \vDash \Sigma_{0} \cup \Sigma_{1}$. Using $\mu$-rc we conclude that $\Sigma_{1} \cup \Sigma$ has a model, hence $T$ has a model.

(ii) Let $\Sigma, \Sigma_{1}$ satisfy the hypothesis required for $\mu$-rc and $L^{*}$ be cf $\mu$-rc. There is $\Sigma_{\alpha}^{\prime} \alpha<\mu$ such that $\cup_{\alpha<\text { cf } \mu} \Sigma_{\alpha}^{\prime}=\Sigma$ and $\left|\Sigma_{\alpha}^{\prime}\right|<\mu$. Now put $\Sigma_{\alpha}=\Sigma_{\alpha}^{\prime}$ $\cup \Sigma_{1}$. Each $\Sigma_{\alpha}(\alpha<\mu)$ has a model, so, by (i), $\cup_{\alpha<\text { cf } \mu} \Sigma_{\alpha}$ has a model.

(iii) is obvious, taking $\Sigma_{1}=\varnothing$.

(iv) easy.

(v) Use (iii) and (iv).

Definition. A cardinal $\kappa$ is weakly characterizable in a logic $L^{*}$ if there is a language $L_{\kappa}$ containing $\{E\}$ and a theory $\Gamma_{\kappa}$ in $L_{\kappa}^{*}$ such that:

(i) there is an expansion $\mathfrak{U}_{\kappa}$ of $\langle\kappa, E\rangle$ satisfying $\Gamma_{\kappa}$.

Let $a_{i}$ be the name of $i \in \kappa$; 
(ii) whenever $\mathfrak{B} \vDash \Gamma_{\kappa} \cup\left\{a_{i} E a_{j}: i<j<\kappa\right\}$ then $\left\{a_{i}: i<\kappa\right\}$ is unbounded in K.

We say that such a $\Gamma_{\kappa}$ weakly characterizes $\kappa$.

LEMMA 6.4. (i) If $\mu$ is regular then $L^{*}$ is $\mu$-rc iff $\langle\mu,\langle\rangle$ is not weakly characterizable in $L^{*}$.

(ii) If $L^{*}$ is $\mu$-rc but not $\lambda$-rc, $\lambda$ regular and $\lambda<\mu$ then there is a uniform ultrafilter $D$ over $\mu$ which is $\lambda$-descendingly complete.

Proof. (i) Assume $L^{*}$ is $\mu$-rc, $\mu$-regular and let $\mathfrak{U}=\langle\mu,\langle, \ldots\rangle$ be any expansion of $\left\langle\mu,\langle\rangle\right.$. Put $\Sigma_{1}$ to be any subset of the $L^{*}$-diagram of $\mathfrak{A}$ and $\Sigma=\{\xi>\alpha \mid \alpha \in \mu\}$ where $\xi$ is a new constant symbol. Clearly $\Sigma$ has cardinality $\mu$ and satisfies the hypothesis of $\mu$-rc. So $\Sigma_{1} \cup \Sigma$ has a model which is not $\mu$-like.

Now assume $L^{*}$ is not $\mu$-rc and let $\Sigma_{1}, \Sigma$ be a counterexample, $\Sigma=\left\{\varphi_{\alpha}\right.$ : $\alpha \in \mu\}, \Sigma_{\beta}=\left\{\varphi_{\alpha}: \alpha<\beta\right\}$. Put $U$ to be a new unary predicate and $E$ a binary relation, say

(a) " $E$ is a linear order on $U$ "; now let $R$ be another new binary relation, say

(b) " $R(x, y) \rightarrow U(x)$ ".

Denote by $\varphi^{R(\xi, x)}$ the relativisation of $\varphi$ to the set $R(\xi, x)$, where $\xi$ acts as a parameter. Say

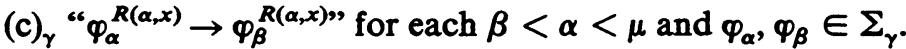

The set $\Sigma_{\gamma}^{\prime}$ defined by (a), (b), (c) $)_{\gamma}$ has the same cardinality as $\gamma$. Put $\Sigma_{1}^{\prime}=\left\{\varphi^{R(\alpha, x)}: \varphi \in \Sigma_{1}, \alpha \in \mu\right\}$.

ClaIM. $\Sigma_{1}^{\prime} \cup \Sigma_{\mu}^{\prime}$ weakly characterizes $\mu$.

First we construct a model. Let $\mathfrak{B}_{\gamma}$ be a model of $\Sigma_{1} \cup \Sigma_{\gamma}$, which exists by assumption. Put $B=\mu \cup \cup{ }_{\gamma<\mu} B_{\gamma}$ where the unions are disjoint. Now put $\mathfrak{B}=\langle B, U, E, R, \ldots\rangle$ where $U^{\mathfrak{P}}=\mu, E$ is the membership of $\mu$ and $R<\cup x_{\gamma<\mu} B_{\gamma}$ such that $R(\gamma, b)$ iff $b \in B_{\gamma}$. The other relations are naturally taken from the $\mathfrak{B}_{\gamma}$. Now let $\mathfrak{R} \vDash \Sigma^{\prime} \cup \Sigma_{\mu}^{\prime} \cup\left\{a_{i} E a_{j}: i<j<\mu\right\}$. Then $\left\{a_{i}\right.$ : $i<\kappa\}$ is unbounded in $U^{\mathfrak{R}}$, for $\Sigma_{1} \cup \Sigma$ has no model.

(ii) Let $\mathfrak{M}$ be a rich and sufficiently large structure such that $H\left(\mu^{+}\right)$is contained in $\mathfrak{M}$. Let $\Sigma_{1}$ be a subset of the $L^{*}$-diagram of $\mathfrak{M}$ weakly characterizing $\left\langle\lambda^{M},\langle\rangle\right.$ and put $\Sigma=\{\alpha<\xi<\mu \mid \alpha \in \mu\}$ where $\xi$ is a new constant symbol. Again $\Sigma$ and $\Sigma_{1}$ satisfy the hypothesis of $\mu$-rc. So there is a model $\mathfrak{R}$ such that $\mathfrak{M}\left\langle\mathfrak{R}\left(L_{\omega \omega}\right)\right.$ and $\mathfrak{N}=\Sigma_{1} \cup \Sigma$. Furthermore we can assume that $\lambda^{M}$ is cofinal in $\lambda^{N}$, using (i). Now define $D$ on $\mu$ by $A \in D$ iff $A \in M$ ( $A$ is a set of $M$ ), $A \subseteq \mu, \bar{A}=\mu$ and $N \vDash \xi \in A^{N}$. Now assume $\left(A_{i}\right)_{i \in \lambda}$ is a descending sequence of sets of $D, A_{0}=\mu$ and $\cap_{i<\lambda} A_{i} \notin D$ (i.e., w.l.o.g. $\bigcap_{i<\lambda} A_{i}=\varnothing$ ). Let $f$ be a function in $\mathfrak{M}$ from $\mu$ into $\lambda$ defined by $f(\alpha)=i$ iff $\alpha \in A_{i}-A_{i+1}$.

Now we have $\mathfrak{R} \vDash \forall \alpha \in \mu(\alpha<\partial \Rightarrow f(\xi)>\alpha)$ since $\mathfrak{R} \xi \xi \in \cap A_{i}$, hence 
we have $\mathfrak{R} \vDash \exists x \forall \alpha \in \mu(\alpha<\partial \Rightarrow f(x)>\alpha)$ and since $\mathfrak{M}<\mathfrak{R} \mathfrak{M} \vDash \exists x \forall \alpha \in \mu$ $(\alpha<\partial \Rightarrow f(x)>\alpha)$. But since $\lambda^{M}$ is cofinal in $\lambda^{N}$, we conclude that $\cap A_{i} \neq$ $\varnothing$, a contradiction.

PROPOSITION 6.5 [KP], [ČC̆]. Let $\mu$ be regular and $D$ be a uniform ultrafilter on $\mu^{+}$. Then

(i) $D$ is $\mu$-descendingly incomplete,

(ii) if $\lambda<\mu, \lambda$ regular and $D$ is $\lambda^{+}$-descendingly incomplete, then $D$ is $\lambda$-descendingly incomplete.

(iii) [JK] Assume $V=L$ (or $\neg 0^{\#}$ or $\neg L^{\mu}$ ). Let $\kappa$ be regular and $D$ be $a$ uniform ultrafilter on $k$. Then $D$ is $\lambda$-descendingly incomplete for all infinite $\lambda$.

Now we can characterize $\mu$-rc.

TheOREM 6.6. (i) $L^{*}$ is $(\kappa, \mu)$-compact for all $\kappa$ and given $\mu$ iff $L^{*}$ is $\kappa$-rc for all $\kappa>\mu$.

(ii) If $L^{*}$ is $\mu^{+}$-rc and $\mu$ is regular, then $L^{*}$ is $\mu-r c$.

(iii) If $\aleph_{\alpha}$ is regular, $n \in \omega$, and $L^{*}$ is $\aleph_{\alpha+n}-r c$, so $L^{*}$ is $\left(\aleph_{\alpha}, \aleph_{\alpha}\right)$-compact.

(iv) $\left(V=L\right.$ or $\neg 0^{*}$ or $\left.\neg L^{\mu}\right)$. If $L^{*}$ is $\mu$-rc then, for all $\lambda<\operatorname{cf}(\mu), L^{*}$ is $\lambda$-rc.

Proof. (i) One direction is trivial. Now assume $L^{*}$ is $\kappa$-rc for all $\kappa>\mu$. We show for each $\lambda>\mu$ that $L^{*}$ is $(\lambda, \mu)$-compact. $\lambda=\mu$ follows from 6.3 (iii), $\lambda>\mu$ from 6.3(iv).

(ii) Assume not, then by 6.4(ii), there is a uniform ultrafilter $D$ on $\mu^{+}$which is $\mu$-descendingly complete, but this contradicts $6.5(\mathrm{i})$.

(iii) Again, assume not; so, by 6.3(iii), $L^{*}$ is not $\aleph_{\alpha}$-rc, so, by 6.6(ii), $L^{*}$ is not $x_{\alpha+n}$-rc for each $n \in \omega$.

(iv) is proved with 6.5(iii) and 6.4(ii).

After this set-theoretic digression we go back to model theory. We shall need an old result of Rabin.

PROPOSITION 6.7 [Ra]. Let $\mu_{0}$ be the first measurable cardinal. For every $\kappa<\mu_{0}$ there is a structure $\mathfrak{A}_{\kappa}=\langle A,<\rangle$ and a language $L^{*}$ such that

(i) $P \in L^{*},\left\langle P^{\mathfrak{x}_{k}},\left\langle\mathfrak{x}^{\mathfrak{x}_{k}}\right\rangle \simeq\langle\omega,\langle\rangle\right.$,

(ii) whenever $\mathfrak{B}\rangle \mathfrak{A}_{\kappa}\left(L_{\omega \omega}^{\kappa}\right)$ then $\left\langle P^{\mathscr{P}},<^{\mathfrak{F}}\right\rangle$ is nonstandard.

Now we are in a position to prove:

THEOREM 6.8. Let $L^{*}$ be a logic such that $\left|L^{*}\right|<\mu_{0}$ for each $L$ with $|L|<\mu_{0}$ and such that $\operatorname{ROB}\left(L^{*}, L^{*}\right)$ and $\mathrm{FV}_{P}\left(L^{*}, L^{*}\right)$ hold. Then $L^{*}$ is $(\omega, \omega)$-compact.

Proof. Let $B_{1}, B_{2}$ be two infinite sets of different cardinality, $\beta_{1}, \beta_{2}$, respectively, such that, for $L=\{=\},\left\langle B_{1}\right\rangle \equiv\left\langle B_{2}\right\rangle\left(L^{*}\right)$.

Since $\left|L^{*}\right|<\mu_{0}$ we may assume that $\beta_{1}, \beta_{2}<\mu_{0}$ using a Hanf-type argument. Now we fix $\kappa>\max \left\{\beta, \beta_{2}\right\}, \kappa<\mu_{0}$. Put $\mathfrak{U}_{\kappa}^{\prime}=\left\langle\mathfrak{U}_{\kappa}, P, Q, a\right\rangle_{a \in \mathfrak{x}_{k}}$ 
where $\mathscr{A}_{\kappa}$ is from 6.7, $P, Q$ are unary and $|P|=\beta_{1},|Q|=\beta_{2}$. Now assume for contradiction $L^{*}$ is not $(\omega, \omega)$-compact. Then $\mathfrak{A}_{\kappa}^{\prime}$ can be expanded to $\mathfrak{A}_{\kappa}^{\prime \prime}$ such that $\mathrm{Th}_{L^{\prime}} \cdot\left(\mathfrak{U}_{k}^{\prime \prime}\right)$ is categorical (using 6.7 and 1.3$)$. Now by $\mathrm{FV}_{P}\left(L^{*}, L^{*}\right)$ we have $\left[\mathfrak{A}_{\kappa}^{\prime \prime}, B_{1}\right] \equiv\left[\mathfrak{A}_{\kappa}^{\prime \prime}, B_{2}\right]\left(L^{*}\right)$. We put now $\left.T=\mathrm{Th}_{L^{\circ}} \cdot \mathfrak{Q}_{\kappa}^{\prime \prime}, B_{1}\right]$. Let $\varphi$ denote the sentence which says that " $F_{1}$ is a bijection from $P^{\mathfrak{x}_{*}^{*}}$ into $B$ (the universe of the second sort)".

Let $\psi$ say " $F_{2}$ is a bijection from $Q^{\mathscr{x}_{*}^{*}}$ into $B$ ". Obviously $T \cup\{\varphi\}$, $T \cup\{\psi\}$ have models $\left[\mathfrak{U}_{\kappa}^{\prime \prime}, B_{1}\right],\left[\mathfrak{\Re}_{\kappa}^{\prime \prime}, B_{2}\right]$, respectively, but $T \cup\{\varphi, \psi\}$ has none since $\beta_{1} \neq \beta_{2}$ and $\mathrm{Th}_{L^{\circ}} \cdot\left(\mathfrak{U}_{\kappa}^{\prime \prime}\right)$ is categorical.

The same proof works under the following hypothesis (cf. 2.15):

COROLlaRY 6.9. If $L^{*}$ is a Karp-logic and $\operatorname{ROB}\left(L_{\omega \omega}, L^{*}\right)$ hold then $L^{*}$ is $(\omega, \omega)$-compact hence $L^{*}=L_{\omega \omega}$ (by 1.2).

PRoof. $\left[\mathfrak{A}_{\kappa}^{\prime \prime}, B_{1}\right] \cong_{P}\left[\mathfrak{A}_{\kappa}^{\prime \prime}, B_{2}\right]$ since $\left\langle B_{1}\right\rangle \cong_{P}\left\langle B_{2}\right\rangle$ and since $L^{*}$ is a Karplogic we have $\left[\mathfrak{U}_{\kappa}^{\prime \prime}, B_{1}\right] \equiv\left[\mathfrak{U}_{\kappa}^{\prime \prime}, B_{2}\right]\left(L^{*}\right)$. Obviously $\varphi_{1}, \varphi_{2}$ are in $L_{\omega \omega}$.

THEOREM 6.10. Let $L^{*}$ be a logic such that $2^{\left|L^{*}\right|}<2^{\alpha_{a+n}}$ whenever $L$ is finite (for some fixed regular $\aleph_{\alpha}$ and $L^{*}$ satisfies $\operatorname{ROB}\left(L^{*}, L^{*}\right)$ and $\mathrm{FV}_{P}\left(L^{*}, L^{*}\right)$ ). Then $L^{*}$ is $\kappa$-rc for each $\kappa>\kappa_{\alpha}$ with $\operatorname{cf}(\kappa)>\kappa_{\alpha}$.

Proof. Assume first $\kappa$ is regular and for contradiction $L^{*}$ is not $\kappa$-rc. So by 6.6(ii) $L^{*}$ is not $\kappa^{+}$-rc. For any $S \subseteq\left\{\beta \mid \beta \in \kappa^{+}\right.$and $\left.\operatorname{cf}(\beta)=\kappa\right\}=C_{\kappa}$ put $\mathfrak{M}_{S}=\left\langle\kappa^{+}, \varepsilon, S\right\rangle$. Assume $\kappa>\aleph_{\alpha+n}$. For $\aleph_{\alpha}<\kappa<\kappa_{\alpha+n}$ we argue with 6.6(ii) iteratedly. Since $2^{\left|L^{\bullet}\right|}<2^{\kappa_{\alpha+n}}$ there are stationary sets $S_{1}, S_{2} \in C_{\kappa}$ with $\left(S_{1}-S_{2}\right) \cup\left(S_{2}-S_{1}\right)=S_{3}$ stationary and $\mathfrak{R}_{S_{1}} \equiv \mathfrak{R}_{S_{2}}\left(L^{*}\right)$. Put now $\mathfrak{A}=$ $\left\langle\kappa^{+}, E, S_{1}, S_{2}, S_{3}, \mathrm{cf}\right\rangle$ with $\operatorname{cf}(\alpha)=\operatorname{cf}(\alpha)$. Since $L^{*}$ is neither $\kappa$-rc nor $\kappa^{+}$-rc we can expand $\mathfrak{A}$ to a structure $\mathfrak{B}_{0}$ and find a theory $\Gamma$ in the expanded language $M$ such that

(i) $\mathfrak{B}_{0} \vDash \Gamma$,

(ii) $\Gamma$ weakly characterizes $\kappa^{+}$,

(iii) $\Gamma$ weakly characterizes $\kappa$,

(iv) for every $\alpha \in \kappa^{+}$with $\operatorname{cf}(\alpha)=\kappa \Gamma$ weakly characterizes $\alpha$.

Put $\mathfrak{O}_{0}^{\prime}=\left\langle\mathfrak{B}_{0}, b \in B\right\rangle$. By $\mathrm{FV}_{P},\left[\mathfrak{P}_{0}^{\prime}, \mathfrak{R}_{S_{1}}\right] \equiv\left[\mathfrak{O}_{0}^{\prime}, \mathfrak{M}_{S_{2}}\right]\left(M_{0}^{*}\right)$ where $M_{0}$ is the appropriate language. Now put $T$ to be the $M_{0}^{*}$-theory of $\left[\mathfrak{O}_{0}^{\prime}, \mathfrak{M}_{\mathcal{S}_{1}}\right]$ and let $\varphi_{i}(i=1,2)$ be the sentence which says " $F_{i}$ is an isomorphism between $\left\langle\left(\kappa^{+}\right)^{\mathbb{P}_{0}}, E^{\mathfrak{O}_{0}}, S_{i}^{\mathbb{B}_{0}}\right\rangle$ and $\mathfrak{M}_{S_{1}}$ " where $F_{1} \neq F_{2}$ both not in $M_{0}$. Obviously $T \cup\left\{\varphi_{i}\right\}$ is satisfiable in $\left[\mathfrak{O}_{0}^{\prime}, \mathfrak{M}_{S_{1}}\right]$.

Clamm 1. $T \cup\left\{\varphi_{1}, \varphi_{2}\right\}$ has no model. Assume, for contradiction, $T \cup$ $\left\{\varphi_{1}, \varphi_{2}\right\}$ has a model $[\mathfrak{B}, \mathfrak{M}]$. Now

$$
\left\langle\left(\kappa^{+}\right), E, S_{1}\right\rangle \simeq_{F_{1}} \mathfrak{R} \simeq_{F_{2}}\left\langle\left(\kappa^{+}\right), E, S_{2}\right\rangle,
$$

put $f=F_{1} \circ F_{2}$. Without loss of generality $f$ is a monotone map $f:\left(\kappa^{+}\right) \rightarrow$ $\left(\kappa^{+}\right)$, for any such isomorphism $g$ is piecewise monotone and a monotone 
map can be constructed from $g$. Now put

$$
A_{0}=\left\{\alpha \in\left(\kappa^{+}\right)^{\mathscr{D}}: \alpha \in \kappa^{+}\right\} \text {and } A_{n+1}=f\left(A_{n}\right)
$$

$A=\cup_{n<\omega} A_{n}$.

Clam 2. $A$ is f-closed, $\langle A, E\rangle$ is a $\kappa^{+}$-like ordering and $A_{0}$ is cofinal in $A$.

Call $X \subseteq A$ c.u.b. if $X \cap A_{0}$ is c.u.b. in $A_{0}$ and $X<A$ stationary if $X$ meets every c.u.b. set in $A$.

Clam 3. $S_{i} \cap A(i=1,2,3)$ are stationary in $A$. Now we distinguish 2 cases:

Case 1. There is a c.u.b set $C \subseteq A$ on which $f$ is the identity. Then $S_{1} \cap A=S_{2} \cap A$ but $A_{0}$ is c.u.b in $A$, hence $A_{0} \cap S_{3} \neq \varnothing$, a contradiction.

Case 2. There is a stationary set $S \subseteq A$ such that $f: S \rightarrow A$ is regressive (otherwise take $f^{-1}$ ). We shall prove an analogue of Fodor's theorem.

Clam 4. There is $\xi \in A$ with $f^{-1}(\xi)$ stationary in $A$. Assume there is no such $\xi$. Then for each $\xi \in A$ there is a c.u.b $C_{\xi} \subseteq A$ such that, for all $\eta \in C_{\xi} \cap S, f(\eta) \neq \xi$. Let

$$
D=\left\{\eta \in A: \forall \xi E \eta\left(\eta \in C_{\xi}\right)\right\}
$$

Then $D \cap S=\varnothing$, so we shall obtain a contradiction if we show that $D$ is c.u.b in $A$. $D$ is clearly closed (i.e., $D \cap A_{0}$ is closed in $A_{0}$ ). Now fix $\gamma \in A_{0}$. Let $\gamma_{0}=\gamma$ and $\gamma_{\alpha^{+}} \in A_{0}$ be some element of $A_{0}$ such that $\gamma_{\alpha} E \gamma_{\alpha^{+}}$and $\gamma_{\alpha^{+}} \in \cap\left\{C_{\xi}: \xi E \gamma_{\alpha}\right\}$ (which is c.u.b in $A$ ). For limits similarly, if $\operatorname{cf}(\alpha)<\kappa$. So we obtain a sequence $\left\{\gamma_{\alpha^{+}}: \alpha \in \kappa\right\}<A_{0}$, which has a supremum $\delta$ in $A_{0}$. By property (iv) of $\Gamma \delta$ is also a supremum in $A$. So we conclude that $\delta \in D$.

This proves Claim 4 and shows that $f$ is not injective, which ends the proof of Claim 1.

If $\kappa$ is singular and $\operatorname{cf}(\kappa) \geqslant \aleph_{\alpha}, L^{*}$ is $\operatorname{cf}(\kappa)$-rc and by Lemma 6.4(ii) $L^{*}$ is $\kappa$-rc.

Piecing this together we now prove Theorem 6.2: The set-theoretic conditions fit into the framework of 6.8 and 6.10. So $L^{*}$ is $\omega$-rc and since we can take $\alpha=0$ in $6.10 L^{*}$ is $\kappa$-rc for each $\kappa>\omega$. With $6.6(i)$ we get the conclusion.

Using 1.6 the condition on the size of $L^{*}$ can be replaced by a suitable Löwenheim-Skolem property. Also Theorems 6.8 and 6.10 can be rephrased with several logics; e.g.:

TheOREM 6.10 (GCH). Assume $L^{\square} \subset L^{*}$ are two logics s.t.

(i) $L^{*}$ satisfies $\mathrm{LS}\left(\aleph_{n}\right)$ for some $n \in \omega$,

(ii) $\mathrm{FV}_{P}\left(L^{\square}, L^{*}\right)$ holds, and

(iii) $\operatorname{ROB}\left(L_{\omega \omega}, L^{\square}\right)$ holds.

Then $L^{\square}$ is $(\kappa, \omega)$-compact for all $\kappa>\omega$. 
THEOREM 6.10". ( $V=L$ or $\left.\neg 0^{\#}\right)$. Assume $L^{\square} \subset L^{*}$ are two logics s.t. (ii) and (iii) from $6.10^{\prime}$ hold, then $L^{\square}$ is $(\kappa, \omega)$-compact for all $\kappa>\omega$.

Proof. Use 6.6(iv).

Corollary 6.11. Assume $L^{*}=L_{\omega \omega}\left[Q^{i}\right]_{i \in \omega}$ and $2^{x_{0}}<2^{k_{n}}$ for some $n \in \omega$. Then if $\operatorname{ROB}\left(L^{*}, L^{*}\right)$ and $\operatorname{FV}_{P}\left(L^{*}, L^{*}\right)$ hold then also $\operatorname{CRAIG}\left(L^{*}, L^{*}\right)$ holds.

Using the following theorem of Lindström [Li3] we get another characterization of $L_{\omega \omega}$.

TheOREM 6.12 (LINDSTRÖM). Assume $L^{*}$ is $(\kappa, \omega)$-compact for each $\kappa$ and $T(L)$ (the union of any $L$-elementary chain is an $L^{*}$-elementary extension of each member of the chain) holds then $L^{*}=L_{\omega \omega}$.

COROLLARY 6.13. $L_{\omega \omega}$ is characterized by

(i) $T(L)$,

(ii) $\operatorname{ROB}(L, L)$,

(iii) $V P(L, L)$,

(iv) $L^{*}=L_{\omega \omega}\left[Q^{1}\right]_{i \in n}$ provided $2^{\mu_{0}}<2^{n_{n}}$ for some $n \in \omega$.

One might ask whether there are proper extensions of $L_{\omega \omega}$ satisfying ROB and $\mathrm{FV}_{p}$. Under the hypothesis of 6.10 such a logic is fully compact and hence satisfies Craig's theorem. In fact we conjecture

CONJECTURE 6.14. Let $L^{*}=L_{\omega \omega}\left(Q^{i}\right)_{i \in \omega}$ be a logic which satisfies ROB and $\mathrm{FV}_{P}$. Then $L^{*}=L_{\omega \omega}$.

If the $Q^{i}$ are all monadic quantifiers, i.e. quantifiers of the form

$$
Q^{i} x_{1}, \ldots, x_{n_{i}}\left(\varphi_{1}\left(x_{1}\right), \varphi_{2}\left(x_{2}\right), \ldots, \varphi_{n_{i}}\left(x_{n_{i}}\right)\right)
$$

then we have:

THEOREM 6.15. Let $L^{*}=L_{\omega \omega}\left(Q^{i}\right)_{i \in \kappa_{*}}$ be a logic with ROB, $\mathrm{FV}_{P}$, each $Q^{i}$ monadic and $\aleph_{\omega}$ strong limit. Then $L^{*}=L_{\omega \omega}$.

Proof. By 6.9 and $6.7 L^{*}$ is $(\kappa, \omega)$-compact for each $\kappa>\omega$. Now let $K$ be a class of monadic structures, i.e. $\mathfrak{A} \in K$ is of the form

$$
\mathfrak{A}=\left\langle A_{1}, \ldots, A_{n} ; P_{1}, \ldots, P_{\kappa}\right\rangle
$$

where $A_{i}$ are universes and $P_{j}$ are unary predicates. Using $\mathrm{FV}_{P}$ we can restrict ourselves to one sorted structures. W.l.o.g. $K$ is a class of cardinals. It suffices to show that $K \in \mathrm{EC}\left(L^{*}\right)$ implies $K \in \mathrm{EC}\left(L_{\omega \omega}\right)$. Now assume there are infinite cardinals $\kappa, \mu$ with $\kappa \in K, \mu \notin K$ and $K \in \operatorname{EC}\left(L^{*}\right)$. Put $T=$ $\mathrm{Th}_{L^{*}}([\kappa, \mu]), T_{1}=\mathrm{Th}_{L^{\bullet}}(\langle\kappa\rangle)$ and $T_{2}=\mathrm{Th}_{L^{*}}(\langle\mu\rangle)$. By compactness $T_{1}$ and $T_{2}$ have arbitrary large models. Let $\varphi_{1}(f)$ say that " $f$ is an injection of the first into the second sort" and $\varphi_{2}(g)$ say that " $g$ is an injection of the second into the first sort". Clearly $T \cup\left\{\varphi_{i}\right\}(i=1,2)$ has a model. For let $\kappa^{\prime}$ be such that 
$\left\langle\kappa^{\prime}\right\rangle \vDash T_{1}$ and $\left.\kappa^{\prime}\right\rangle \mu$, so $\left[\kappa^{\prime}, \mu\right]$ can be expanded to satisfy $\varphi_{1}$ and, by $F V_{P}$, $\left[\kappa^{\prime}, \mu\right] \vDash T$ and similarily for $\varphi_{2}$. But then by ROB $T \cup\left\{\varphi_{1}, \varphi_{2}\right\}$ has a model $\left[\kappa^{\prime \prime}, \mu^{\prime \prime}\right]$ so by the Cantor-Bernstein Theorem $\kappa^{\prime \prime}=\mu^{\prime \prime}$. But by $\mathrm{FV}_{P}, \kappa^{\prime \prime} \in K$, $\mu^{\prime \prime} \notin K$ a contradiction. So w.l.o.g. $K$ contains only finite cardinals, hence, by compactness, the cardinals in $K$ are bounded by some natural number $n \in \omega$ and $K \in \operatorname{EC}\left(L_{\omega \omega}\right)$.

The argument above can still be extended (cf. [Ma5]). Let $K$ be a class of structures $\mathfrak{A}$ of the form $\left\langle A, E, P_{1}, \ldots, P_{n}\right\rangle$ where $A$ is the universe, $E$ is an equivalence relation and $P_{i}$ are monadic predicates. If $K$ is closed under isomorphism and $K \in \mathrm{EC}\left(L^{*}\right)$ for $L^{*}=L_{\omega \omega}\left(Q^{i}\right)_{i<\kappa_{\omega}}$, the $Q^{i}$ arbitrary quantifiers, $L^{*}$ satisfies $\mathrm{ROB}$ and $\mathrm{FV}_{P}$ and $\aleph_{\omega}$ strong limit, then $K \in$ $\operatorname{EC}\left(L_{\omega \omega}\right)$. Here we use that two equivalence relations with $\kappa$ equivalence classes, each of cardinarlity $\kappa$, are isomorphic.

This proves Conjecture 6.14 for some special classes of quantifiers. The problem remains of how to extend the above proof to arbitrary quantifiers.

Note that Caicedo [Ca] got a weak version of 6.15 without set-theoretic assumptions and $\mathrm{FV}_{P}$ but using ( $\left.\omega, \omega\right)$-compactness (cf. [Ma5]).

7. The definability of the syntax-structure. In this section we present without proofs two abstract theorems due to Burgess and Paulos on the weak Beth-property. We follow [Fe2] for terminology.

THEOREM 7.1 (BURGESs). Let $L^{*}$ be an absolute, normal logic, $L^{*} \subseteq H\left(\omega_{1}\right)$ such that WBETH $\left(L^{*}, L^{*}\right)$ holds. Then the class of countable well-orderings is not $\mathrm{PC}\left(L^{*}\right)$.

TheOREM 7.2 (Paulos). Let $L^{\Phi} \subset L^{\square}$ be two logics such that $L^{\Phi}$ is adequate to truth for $L^{\square}$ and WBETH( $\left.L^{\Phi}, L^{\square}\right)$ holds, then no syntax-structure of $L^{\square}$ is

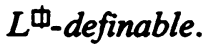

Theorem 7.1 is a slight generalization of Paulos' theorem [Pa]. The main reason we quote these theorems here is that combining the methods of proofs together with 6.1 Gostanian and Hrbacek [GH] produced uniformly rather tricky counterexamples to WBETH $\left(L^{*}, L^{\square}\right)$ for

$$
\begin{aligned}
& L^{*}=L_{\omega, \omega}^{G}, L^{\square}=L_{\infty \infty}^{G}, \\
& L^{*}=L_{\omega, \omega}^{G}, L^{\square}=L_{\infty \infty}, \\
& L^{*}=L_{\kappa^{+} \omega}, L^{\square}=L_{\lambda \lambda} \text { for all regular } \lambda \text { which are } L_{\kappa^{+} \omega} \text { accessible. }
\end{aligned}
$$

8. Some direct applications. In this section we present some applications of the abstract theory. Less direct applications may be found in [MS2], [MS3]. The table below shows how much can be drawn from the abstract approach.

Most of the notation of the table is self-evident. $L_{\omega_{1} \omega}^{G}$ is the logic obtained from $L_{\omega_{1} \omega}$ adding the game quantifier. $L^{H}$ is the logic obtained from $L_{\omega \omega}$ adding the Henkin quantifier. In fact any nonlinear partially ordered quantifier will lead to the same situation (cf. [Wa]). 


\begin{tabular}{|c|c|c|c|c|c|}
\hline & CRAIG & ROB & BETH & WBETH & $\mathrm{WFV}_{P}$ \\
\hline$L_{\omega \omega}$ & yes [Ke2] & yes [Ke2] & yes & yes & yes \\
\hline $\begin{array}{l}L_{\omega_{1} \omega} \omega\left(L_{A}\right) \\
A \text { countable }\end{array}$ & $\begin{array}{l}\text { yes [Ke2] } \\
\text { [LE] }\end{array}$ & $\begin{array}{l}\text { no } 2.14 \\
\text { (but yes for } \\
\text { countable } T \text { ) }\end{array}$ & yes & yes & no 3.2 \\
\hline$L_{\omega_{1} \omega}^{G}$ & no $[\mathbf{G H}]$ & $\begin{array}{l}\text { no } 2.14 \\
\text { yes for } \\
\text { countable } T\end{array}$ & no $[\mathbf{G H}]$ & no $[\mathbf{G H}]$ & no 3.4 \\
\hline$\underline{L_{k}+\omega} k \geqslant \omega_{1}$ & no 2.12 & $\begin{array}{l}\text { no } 2.14 \\
\text { or } 6\end{array}$ & no 2.12 & $\begin{array}{l}\text { no } 3.4 \\
{[\mathrm{GH}] \operatorname{cf}(k)>\omega}\end{array}$ & no 3.4 \\
\hline$L\left(Q_{\kappa}\right) \quad k \geqslant \omega_{1}$ & no $[\mathrm{Fe}]$ & $\begin{array}{l}\text { no } \\
\text { (compactness) }\end{array}$ & $\begin{array}{l}\text { no } 5.4 \\
\text { and }\left[W_{0}\right]\end{array}$ & $?$ & yes [Wo] \\
\hline$L\left(Q_{k}\right) \quad k=\omega$ & $\begin{array}{r}\text { no } 3.2 \\
2.3 \\
\end{array}$ & no 2.15 & $\begin{array}{r}\text { no } 3.2 \\
2.3 \\
\end{array}$ & no 3.2 & yes [Wo] \\
\hline$L^{H}$ & $\begin{array}{r}\text { no } 6.1 \\
2.3\end{array}$ & $\begin{array}{ll}\text { no } 1.5 \\
& 2.14 \\
\text { or } 6.1 \\
\end{array}$ & $\begin{array}{r}\text { no } 6.1 \\
2.3\end{array}$ & no 6.1 & no 8.1 \\
\hline$L^{P}, L(a a)$ & no [MS2] & no (comp) & no [MS2] & $?$ & $\begin{array}{l}\text { yes } L^{P} \\
\text { no } L(a a)\end{array}$ \\
\hline$L\left(Q^{<n}\right)$ & no [Bd] & no (comp) & no 5.4 & $?$ & yes [Bd] \\
\hline$L^{n}$ & no 5.4 & no 6.2 & no 5.4 & no 6.1 & yes [MS2] \\
\hline$L^{\prime \prime}$ & no 7.2 & no 6.2 & no 7.2 & no 7.2 & no 8.1 \\
\hline
\end{tabular}

Finally $L^{P}$ is positive logic and $L(a a)$ stationary logic (cf. [BKM], [M24] and [MS2]). $L\left(Q^{<n}\right)$ is the Magidor-Malitz logic from [MM], $L^{n}$ is negative logic (cf. [BKM], [MS2]) and $L^{\mathrm{II}}$ is second order logic.

To make the table below more complete let us finish with a last observation.

Let $\operatorname{Exy}(\phi(x), \psi(y))$ be a binary quantifier binding two formulas and expressing that $\phi$ and $\psi$ define sets of the same cardinality.

Propostrion 8.1. Let $L^{*}$ be a logic which is a set for each $L$ and which extends $L_{\omega \omega}(E)$. Then $\operatorname{WFV}_{P}\left(L_{\omega \omega}(E), L^{*}\right)$ fails.

Examples for such $L$ are $L^{H}$ or $L^{\text {II }}$ (cf. [KL]).

Proof. Let $A, B$ be two sets of different cardinality such that $A \equiv B\left(L^{*}\right)$. Such sets exist by a Hanf type argument. But $[A, B] \neq[A, A]\left(L^{*}\right)$ using the quantifier $E$.

ADDED IN PROOF (June 25, 1979). If we redefine ROB by allowing arbitrary theories $T_{1}, T_{2}$ over arbitrary sets of predicates $\bar{P}$ instead of $\varphi(P), \psi(P)$, the results of this section can be improved.

THEOREM. Under the same set-theoretic hypothesis as in 6.10 if $L^{*}$ satisfies ROB, then $L^{*}$ is $\kappa$-rc for each $\kappa>\aleph_{\alpha}$.

In the forthcoming paper by the same authors the amalgamation property of logics will be studied. We shall show that under much weaker assumptions than in 6.10 the amalgamation property implies full compactness. This will 
give us also some answers to Problem 2 in the introduction to this paper. The above version of ROB was suggested to us by a preprint of $D$. Mundici.

\section{REFERENCES}

[Am] Y. Amihai, "The two of us together ..." in The modern Hebrew Poem itself (Burnshaw etal., Editors), New York, 1966:

[Ba1] K. J. Barwise, Axioms for abstract modeltheory, Ann. Math. Logic 7 (1974), 221-265.

[B22] __, Admissible sets and structures, Perspectives in Math. Logic, vol. 1, SpringerVerlag, Heidelberg, 1975.

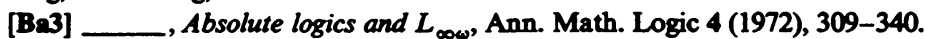

[Ba4] __ Some applications of Henkin quantifiers, Israel J. Math. 25 (1976), 47-63.

[BKM] K. J. Barwise, M. Kaufmann and M. Makkai, Stationary logic, Ann. Math. Logic 13 (1978), 171-224.

[Bd] L. Badger, An Ehrenfeucht game for the multivariable quantifiers of Malitz and some applications, Pacific J. Math. 72 (1977), 293-304.

[BS] J. Bell and H. Slomson, Models and ultraproducts, North-Holland, Amsterdam, 1969.

[B1] V. V. Bludov, Uniquely orderable groups, Algebra i Logika 13 (1975), 609-634.

[Bu] J. P. Burgess, "Descriptive set theory and infinitary languages," in Set theory. Foundations of mathematics (Proc. Sympos., Belgrade, 1977), Institute Mathématique, Recueil des Travaux, Nouvelle Série, tome 2 (10), 1977, pp. 9-30.

[Ca] X. Caicedo, Maximality and interpolation in abstract logics, Thesis, University of Maryland, 1978.

[CK] C. C. Chang and J. K. Keisler, Model theory, 2nd ed., North-Holland, Amsterdam, 1977.

[CC] G. V. Cudnovskiı and D. V. Cudnovskï, Regularnye $i$ ubyoajušce nepolnye ultrafil'try, Dokl. Akad. Nauk SSSR 198 (1971), 779-782.

[Fe1] S. Feferman, Applications of many-sorted interpolation theorems, Proc. Sympos. Pure Math., vol. 25, Amer. Math. Soc., Providence, R. I., 1971, pp. 205-224.

[Fe2] _ , Two notes on abstract model theory. I, II, Fund. Math. 82 (1974), 153-165; ibid 89 (1975), 111-130.

[F1] J. Flum, First order logic and its extensions, Proc. Logic Conference, Kiel 1974, Lecture Notes in Math., vol. 499, Springer-Verlag, Berlin and New York, 1976, pp. 248-310.

[Fr] H. Friedman, Beth's theorem in cardinality logics, Israel J. Math. 14 (1973), 205-212.

[GH] R. Gostanian and K. Hrbacek, On the failure of the weak Beth property, Proc. Amer. Math. Soc. 58 (1976), 245-249.

[Gr] J. Gregory, Beth definability in infinitary languages, J. Symbolic Logic 39 (1974), 22-26.

[Ha] L. Harrington, Extensions of countable infinitary logic which preserve most of its nice properties, Arch. Math. Logik Grundlagenforsch. (to appear).

[JK] R. B. Jensen and B. J. Koppelberg, A note on ultrafilters, Notices Amer. Math. Soc. 25 (1978), p. A-250. Abstract 78T-E21.

[Ke1] J. Keisler, Logic with the quantifier "there exist uncountably many", Ann. Math. Logic 1 (1969), 1-93.

[Ke2] __, Modeltheory for infinitary logic, North-Holland, Amsterdam, 1971.

[KI] S. C. Kleene, Finite axiomatizability of theories in the predicate calculus using additional predicate symbols, Mem. Amer. Math. Soc. No. 20 (1952), 27-68.

[KL] M. Krynicki and A. Lachlan, On the semantics of the Henkin quantifier (preprint).

[Kue] D. Kueker, Countable approximations and Löwenheim-Skolem theorems, Ann. Math. Logic 11 (1977), 50-103.

[KP] K. Kunen and K. Prikry, Descendingly incomplete ultrafilters, J. Symbolic Logic 36 (1971), 650-652.

[u1] P. Lindström, First order logic and generalized quantifiers, Theoria 32 (1966), 187-195.

[Li2] _ On extensions of elementary logic, Theoria 35 (1969), 1-11.

[LB] "A characterization of elementary logic," in Modality, morality and other problems of sense and nonsense, Essays dedicated to S. Halden, Reidel, Lund, 1973, pp. 189-191. 
[Le] E. G. K. Lopez-Escobar, An interpolation theorem for denumerably long formulas, Fund. Math. 57 (1965), 254-272.

[MM] M. Magidor and J. Malitz, Compact extensions of $L(Q)$, Ann. Math. Logic 11 (1977), 217-261.

[MI] J. Malitz, Infinitary analogues of theorems from first order model theory, J. Symbolic Logic 36 (1971), 216-228.

[Ma1] J. A. Makowsky, Securable quantifiers, $k$-unions and admissible sets, Logic Colloquium 73, North-Holland, Amsterdam, 1975, pp. 409-428.

[Mr2] Some observations on uniform rechuction for properties invariant on the range of definable relations, Fund. Math. 99 (1978), 129-133.

[Ma3] __ Die Modelltheorie oon $L^{P}$, in preparation.

[Ma4] __, Quantifying over countable sets: pasitive us. stationary logic, Logic Colloquium 77 (A. Macintyre et al., Editors), North-Holland, Amsterdam, 1978, pp. 183-194.

[Ma5] __ Characterizing monadic and equivalence quantifiers, in preparation.

[MS2] J. A. Makowsky and S. Shelah, The theorems of Beth and Craig in abstract Modeltheory. II, Compact logics, Arch. Math. Logik Grundlagenforsch. 20 (1979), no. 1/2.

[MS3] _ The theorems of Beth and Craig in abstract Modeltheory. III, Infinitary-and $\Delta$-logic, in preparation.

[MSS] J. A. Makowsky, S. Shelah and J. Stavi, $\Delta$-logics and generalized quantifiers, Ann. Math. Logic 10 (1976), 155-192.

[Na] M. Nadel, An arbitrary equivalence relation as elementary equivalence in an abstract logic, Z. Math. Logik Grundlagen Math. (to appear).

[NSt] M. Nadel and J. Stavi, $L_{\infty \lambda}$-equivalence, isomorphisms and pretential isomorphism, Trans. Amer. Math. Soc. 236 (1978), 51-74.

[Pa] J. Paulos, Noncharacterizability of the syntax set, J. Symbolic Logic 41 (1976), 368-372.

[Ra] M. O. Rabin, Arithmetical extensions with prescribed cardinality, Indag. Math. 21 (1959), 439-446.

[Sh1] S. Shelah, Refuting Ehrenfeucht's conjecture, Israel J. Math. 25 (1976), 273-286.

[Sh2] __, On models with power like orderings, J. Symbolic Logic 37 (1972), 247-267.

[Sh3] __, Generalized quantifiers and compact logic, Trans. Amer. Math. Soc. 204 (1975), 342-364.

[St1] J. Stavi, unpublished manuscript.

[St2] _ Compactness properties of infinitary and abstract languages. I, Logic Colloquium 77 (A. Macintyre et al., Editors), North-Holland, Amsterdam, 1978, pp. 263-276.

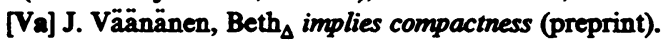

[Wa] W. J. Walkoe, Jr., Finite partially-ordered quantification, J. Symbolic Logic 35 (1970), 535-555.

[Wo] A. Wojciechewska, Generalized products for $Q_{\alpha}$-languages, Bull. Acad. Polon. Sci. Sér. Sci. Math. Astronom. Phys. 17 (1969), 337-339.

II. Mathematisches Institut, Freie Universträt Berin, 1 Berinn 33, West Germany

Department of Mathematics, The Hebrew Universtty, Jerusalem, Israel 